\title{
Advances in organic micro/nanocrystals with tunable physicochemical properties
}

\author{
Yingxin $\mathrm{Ma}^{1 \dagger}$, Zhi-Zhou $\mathrm{Li}^{2 \dagger}$, Hongtao Lin ${ }^{1^{*}}$, Shuhai Chen ${ }^{1 *}$, Shuping Zhuo ${ }^{1}$ and Xue-Dong Wang ${ }^{2 *}$
}

\begin{abstract}
Organic micro/nanocrystals based on small organic molecules have drawn extensive attention due to their potential application in organic field-effect transistors, electrochemical sensors, solar cells, etc. Herein, the recent advances for organic micro/nanocrystals from the perspective of molecule aggregation mode, morphology modulation, and optical property modulation are reviewed. The stacking mode and the intermolecular interaction depend on the molecular structure, which eventually determines the morphology of organic micro/nanocrystals. The morphologies of the organic micro/nanocrystals make the aggregates exhibit photon confinement or light-guiding properties as organic miniaturized optoelectronic devices. In this review, we conclude with a summary and put forward our perspective on the current challenges and the future development of morphology and optical tunable direction for the organic micro/nanocrystals.
\end{abstract}

Keywords: organic micro/nanostructures, organic semiconductor, aggregation mode, morphology modulation, excitedstate process

\section{INTRODUCTION}

Organic micro/nanocrystals have been demonstrated as brilliant building-blocks for the miniaturized devices [1-3], electrochemical sensors [4-7], organic light-emitting diodes (OLEDs) $[8,9]$, solar cells $[10,11]$, and organic field-effect transistors $[12,13]$, and thus have drawn enormous attention from researchers [14]. Compared with the inorganic materials, which occupy strong market positions and accessibility to the manufacturing infrastructure, the organic counterparts play a unique role due to the broad spectral tunability, high optical crosssections as well as low-cost solution processing [15-17]. What's more, the periodic molecular packing mode in the micro/ nanocrystals leads to a higher mobility and regular morphology, which make them distinguish from the amorphous structures and polymers [18-20]. The crystal's morphology significantly affects the final performance of solid products, such as the bioavailability for pharmaceutical compounds, reactivity for catalysts, filtering and drying times [21-23]. As for the optoelectronics, the morphologies of the micro/nanocrystals seriously affect the performance of devices, such as the propagation modes in waveguide devices and the resonant modes in the organic solid-state lasers (OSSLs) [24-27]. Many studies have focused on the shape modulation of the organic micro/nanocrystals, trying to improve the controllability and pre-design ability $[28,29]$. Nevertheless, it remains a huge challenge due to the inadequate understanding of the basic growth mechanisms, limited preparation methods, and the elusive influence of various factors, such as additives, supersaturation, temperature, and solvents. Besides that, different from inorganic materials, the controllable growth process of organic micro/nanocrystals is much more complicated due to the weak intermolecular forces, such as hydrogen/halogen bonds, charge-transfer (CT) interaction, $\pi-\pi$ interaction, and van der Waals force, which lead to a recondite self-assembly process [30-33].

The optical properties of the organic micro/nanocrystals, such as the emission color, emission efficiency, and the fluorescent/ phosphorescent lifetime, fall in the core requirements during the preparation of high-efficiency optoelectronic devices [34,35]. To modulate the optical properties, the molecular structures are usually redesigned or modified by extra functional groups, which is demonstrated as a powerful pathway to adjust the optical properties and other physicochemical properties [36,37]. But it is still challenging to modulate the properties of the molecular crystals yet not changing the structure of molecules.

Herein, we review the advances in the progress of modulation routes on the morphologies and optical properties of the organic micro/nanocrystals, which were fabricated by small molecules. The modulation methods of the molecular aggregation modes were firstly introduced for the significant effect on the morphology and optical properties of the final crystals. Then the controlling approaches for the morphology and optical properties (mainly for the emission wavelength, efficiency, lifetime and so on) were summarized for the core function in the optoelectronic systems. Lastly, we put forward an outlook on the future applications of the organic micro/nanocrystals with different shapes and tunable optical properties.

\section{AGGREGATION MODES}

In contrast to the inorganic materials composed of strong chemical bonding, the organic counterparts are supported by means of weaker intermolecular forces, which means the molecular packing modes can significantly influence the morphology and optical properties of micro/nanocrystals, so as to be employed as an important method in various fields, such as catalysts, solar

\footnotetext{
${ }^{1}$ School of Chemistry and Chemical Engineering, Shandong University of Technology, Zibo 255000, China

${ }^{2}$ Jiangsu Key Laboratory for Carbon-Based Functional Materials \& Devices, Institute of Functional Nano \& Soft Materials (FUNSOM), Soochow University, Suzhou 215123, China

$\dagger$ These authors contributed equally to this work.

* Corresponding authors (emails: wangxuedong@suda.edu.cn (Wang XD); linht@sdut.edu.cn (Lin H); shuhaichen@sdut.edu.cn (Chen S))
} 
cells, and the photo-responsive switch [38,39]. More than 50 years ago, Kasha [40-42] established a relationship between the geometry of the stacking and the photophysical property. In his model, H-aggregates and J-aggregates are formed from the separated monomer molecular dimers stacked "side-by-side" and "head-to-tail", respectively; the former exhibits blue-shifted absorption maximum suppressing radiation attenuation rate, while the latter exhibits a red-shifted absorption maximum enhancing radiation attenuation rate (Fig. 1a). In recent years, a novel mechanism for molecular aggregates is presented based on wave function overlap (WFO) coupling supplementing Coulombic coupling in the traditional Kasha model. The Coulombic coupling between any two chromophores is caused by the interaction between their transition charge distributions usually expressed by the point-dipole approximation $[43,44]$ :

$J_{\text {coul }} \approx \frac{\mu^{2}\left(1-3 \cos ^{2} \theta\right)}{4 \pi \varepsilon R^{3}}$,

where $\mu$ is the transition dipole moment (TDM), $R$ represents the intermolecular distance between mass centers, $\theta$ is the slip angle between $\mu$ and $R$, and $\varepsilon$ is the optical dielectric constant of the medium (see Fig. 1b). $\theta$ value is the key factor to distinguish
$\mathrm{H}$ - and J-aggregates: if $1-3 \cos ^{2} \theta>0, J_{\text {coul }}>0$, which indicates the $\mathrm{H}$-aggregate mode; while if $1-3 \cos ^{2} \theta<0, J_{\text {coul }}>0$, which indicates the J-aggregate mode. In tightly packed molecular aggregates, an additional short-range CT-mediated exciton coupling can be comparable to the long-range Coulombic coupling, which results in WFO-coupled J-/H-aggregates (Fig. 1c).

What's more, by several excellent examples, through the small longitudinal or lateral displacements of the molecular dimers, the significant effect of molecular aggregation mode on molecular energy has also been found $[45,46]$. For example, as shown in Fig. 1d, a quantum-chemistry-based protocol was proposed by Engels and his colleagues [46] to obtain the potential energy surfaces of perylene bisimide (PBI) dimers for ground and excited states; therefore, the spectral properties can be well calculated further. When only transitional offsets were considered, two distinct minima were found corresponding to the redcolored dyes and black-colored dyes of majority of the PBI crystals with different substituents, which suggest the existence of H-/J-type exciton coupling, respectively. By these brilliant calculations, the transformation between the molecular aggregate modes is proved to be possible by proper modulation.

To date, among the amounts of examples with different
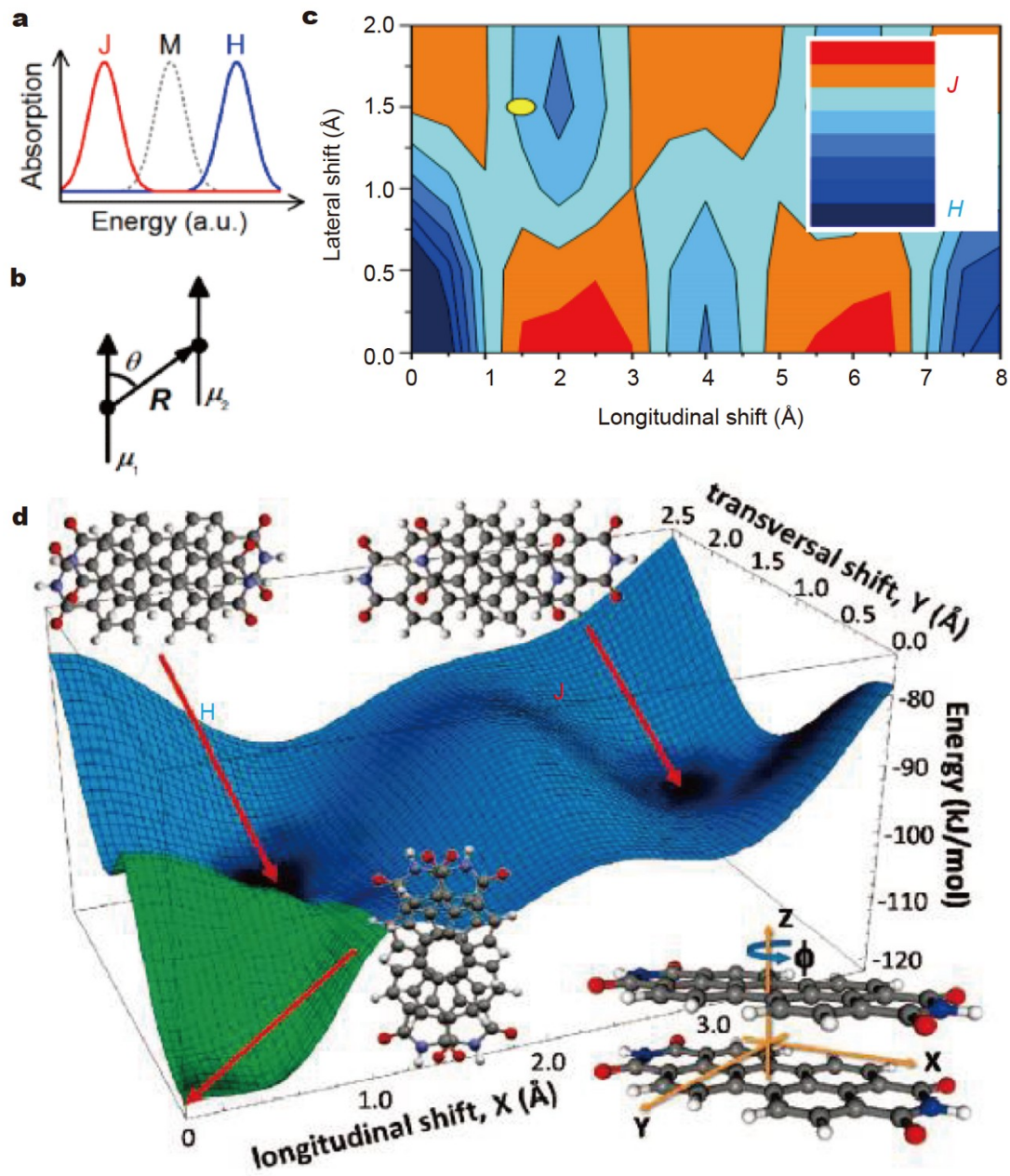

Figure 1 (a) Ideal absorption spectra of J-/H-aggregates based on Kasha exciton energy bands. (b) The Coulombic coupling transition dipole geometry is calculated under the point dipole approximation. Reproduced with permission from Ref. [43]. Copyright 2017, American Chemical Society. (c) Calculated values of JWFO (in $\mathrm{cm}^{-1}$ ) between two 8T's in $C_{i}$ dimer as a function of longitudinal and lateral displacements. Reproduced with permission from Ref. [44]. Copyright 2012, American Chemical Society. (d) Calculated potential energy surface of the ground state. Reproduced with permission from Ref. [46]. Copyright 2008, American Chemical Society. 
molecular aggregates, the perylene derivatives are especially interesting for that the $\mathrm{H}$-aggregates and J-aggregates have both been found among them [47-51]. The first case of the transformation from the $\mathrm{H}$-aggregates to J-aggregates was achieved based on the perylene derivative, PBI, by Würthner and colleagues [48]. In their study, the transformation can be achieved by two approaches: the complexation reaction and the temperature-dependent effect. When $\mathrm{PBI} / \mathrm{dCA}=1: 0.5$, where $\mathrm{dCA}$ represents the monosubstituted cyanurate ( $\mathrm{d}$ denotes single dodecyl group), the absorption of the free perylene chromophore completely disappeared, indicating that an $\mathrm{H}$-dimer was formed quantitatively through the synergistic effect of hydrogen bonding interaction and $\pi$-stacking. However, as they made the mixture solution of $\mathrm{PBI} / \mathrm{dCA}=1: 1$, interestingly, red solution turns to green color, and meanwhile, a new absorption band appears at $620 \mathrm{~nm}$ and the $\mathrm{H}$-dimer decreases. Compared with the absorption of monomer PBI $(515 \mathrm{~nm})$, the new band is redshifted by $>100 \mathrm{~nm}$, which indicates the J-type aggregates formed in the solution (Fig. 2a). J-aggregates can also be converted to $\mathrm{H}$-aggregates verified by the temperature-dependent ultraviolet-visible (UV-Vis) measurements (Fig. 2b). With the progress of increasing the temperature, the spectral transition in two nearly independent stages occurs with clear isosbestic points at 554 and $523 \mathrm{~nm}$ (purple and blue arrows depicted in Fig. 2b, with temperature of $20-55^{\circ} \mathrm{C}$ and $60-90^{\circ} \mathrm{C}$ respectively). Fig. $2 \mathrm{c}$ indicates that the temperature of $55^{\circ} \mathrm{C}$ is the inflection point, and below $55^{\circ} \mathrm{C}$, primarily the transformation from $\mathrm{J}$ - to $\mathrm{H}$ aggregates occurs, which can be confirmed by the decrease of $\varepsilon$ value at 400 and $620 \mathrm{~nm}$. When the temperature exceeds $55^{\circ} \mathrm{C}$, according to the change of $\varepsilon$ value at $544 \mathrm{~nm}$, it can be concluded that only the conversion of $\mathrm{H}$-aggregate to monomer takes place. The aggregation transformation in the PBI in the liquid-crystalline state was then supplemented by Asha's group [30] who synthesized a sequence of strong fluorescent PBI molecules with different terminal phenyl substituents. This work proved the effect of the substitution on the aggregation
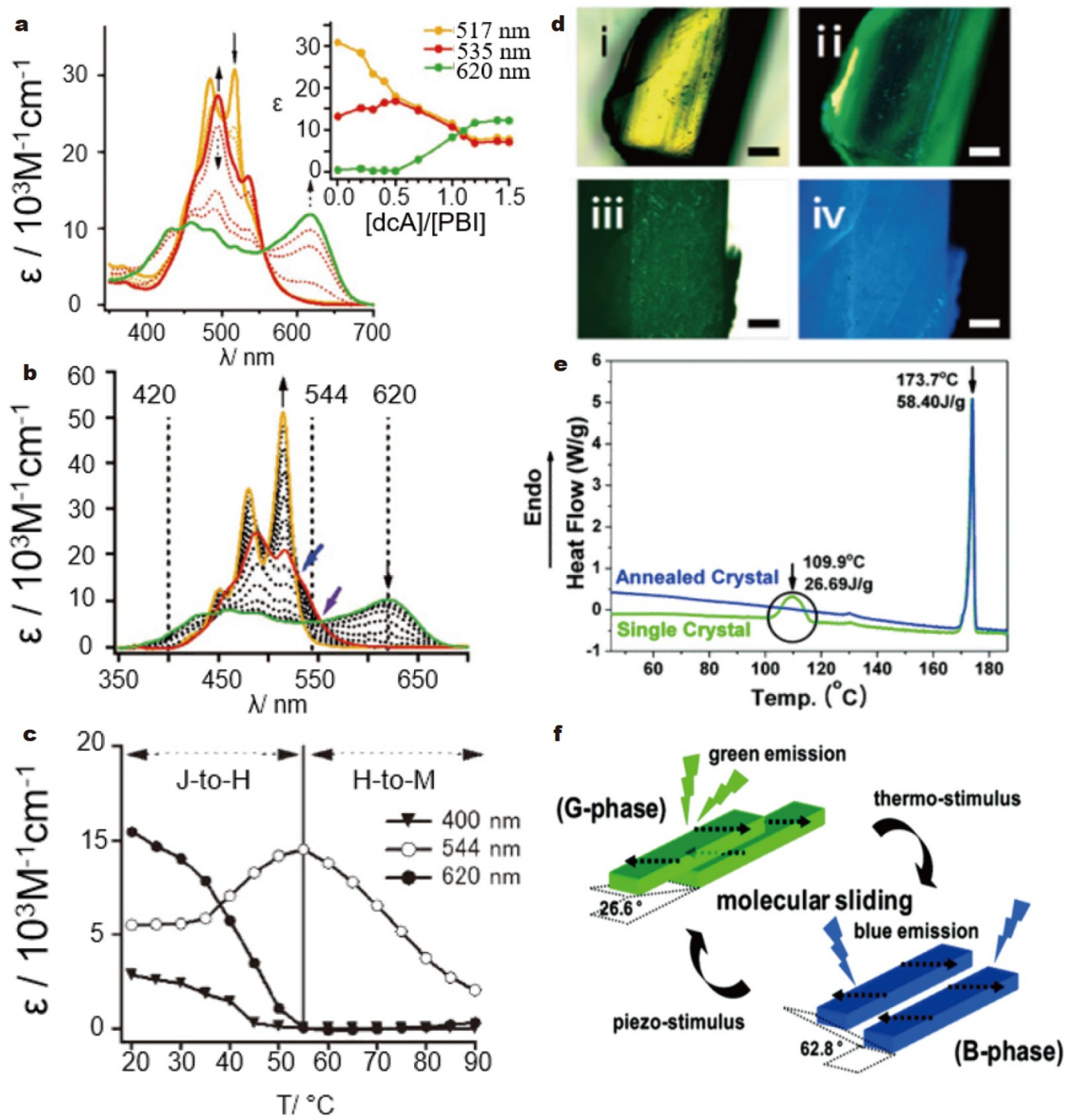

Figure 2 (a) UV-Vis of PBI/dCA with different complexation reactions. Inset shows the changes of $\varepsilon$ at several wavelengths. (b) Temperature-dependent UV-Vis spectra of PBI/dCA. (c) Plots of $\varepsilon$ versus temperature at 400, 544 and $620 \mathrm{~nm}$. Reproduced with permission from Ref. [48]. Copyright 2008, Wiley. (d) Photos of the single crystal before annealing, under (i) room light, (ii) UV light, and after annealing, (iii) under room light, and (iv) UV light with the scale bar of $0.2 \mathrm{~mm}$. (e) DSC trace of the single crystal and annealed crystal. (f) Schematic diagram of slip-stacking modes in DBDCS sheets. Reproduced with permission from Ref. [52]. Copyright 2010, American Chemical Society. 
geometry, which drives the formation of different packing modes, thus leading to the significant modulation in the emission and absorbance bands. The aggregation-mode transformation of the organic crystals has also been demonstrated along with the change of the morphology and optical properties.

As another excellent example, Yoon et al. [52] demonstrated multi-stimuli two-color luminescence in a cyanostilbene-based molecule, (2Z,2'Z)-2,2'-(1,4-phenylene)bis(3-(4-butoxyphenyl) acrylonitrile) (DBDCS), attributed to the change in the molecular aggregate modes. The prepared DBDCS crystal is yellow under UV light and emits green light, which is called G-phase. Nevertheless, if the sample is heated to $125^{\circ} \mathrm{C}$ for $1 \mathrm{~h}$, the color turns to pale green and the emission turns to blue, which is called B-phase (Fig. 2d). Indeed, under $125^{\circ} \mathrm{C}$ annealing conditions, this crystalline-state transition with characteristic fluorescence change is associated with the first-order endothermic peak in the differential scanning calorimetry (DSC) thermogram (Fig. 2e). Moreover, the parallel molecular staking of the two phases has different slip angles of $26.6^{\circ}$ and $62.8^{\circ}$, respectively; according to the formula mentioned above, it can be concluded that G-phase and B-phase are J-aggregates and H-aggregates, respectively (Fig. 2f).

Likewise, Yang et al. [53] demonstrated that despite the same chemical structure, the crystal properties are highly related to the molecular configuration and packing mode. They reported three crystalline polymorphs of 4 - $(4 a, 10 a$-dihydro- $10 H$-phenothiazin10-yl)benzonitrile (CzS-CN), which display much different properties of room-temperature phosphorescence (RTP) and aggregation-induced emission (AIE). Due to their different molecular packings, the RTP lifetimes change from 266 to $41 \mathrm{~ms}$, and then to $32 \mathrm{~ms}$, accompanied by different photoluminescence quantum yields (PLQYs) of crystals from $22.6 \%$ and $17.8 \%$ to $6.9 \%$. In addition, Wang and his colleagues [54] achieved RTP and mechanoluminescence $(\mathrm{ML})$ in polymorphisms of a triphenylamine derivative with ortho-substitution. This molecular packing-dependent emission affords crucial information to deeply understand the intrinsic mechanism of different emission forms and the possible packing-function relationship.

Thus, the molecular aggregate mode is the underlying reason for the physicochemical properties of the organic micro/nanocrystals, which can significantly influence the optical properties and the morphology [55]. Other modulation methods for the morphology and optical properties of the organic crystals without changing the molecular packing modes will be discussed in the following sections.

\section{MORPHOLOGY MODULATION}

The uniform shape and tunable optical properties of the organic micro/nanocrystals, and their capability to be integrated on a large scale to a flexible substrate are extremely important for their application in OSSLs [25], LEDs [56,57], field-effect transistors [58], solar cells [59], and so on. The desirable optoelectronic performances mainly lie on the structure of the molecules and the intermolecular interactions such as the molecular arrangement mode or packing in the condensed states. The molecular packing mode can be modulated by the weak intermolecular interactions such as CT interaction, $\pi-\pi$ stacking, van der Waals force, and hydrogen bonding, which induce photon confinement or light-guiding performances and finally influence the optical/electrical properties of the organic micro/nanocrystals [30]. For example, a high-degree order of molecule stacking mode is conducive to the anisotropic charge transfer in organic field-effect transistors [60]. In addition, the regular morphology of the organic micro/nanocrystals is conducive to the favorable medium and optical resonators for organic lasers [61].

Previously, researchers reported a solvent evaporation method that the weak noncovalent interactions induce organic molecules bound together to form microcrystals with good morphology when the solvent evaporates [62]. This method can achieve lowcost large-area fabrication for organic-molecule semiconductors, but faces the challenge of solvent corroding and dissolvation of the organic crystals. Consequently, in order to improve the solvent-resistant capacity, Lan et al. [63] put forward a solventresistant coating method. The coatings involved layer-by-layer assembly of poly(diallyldimethylammonium) (PDDA) and poly (styrene-sulfonate) (PSS) onto the crystals, followed by immersing the coated crystals into polyvinyl alcohol (PVA) aqueous solutions for $2 \mathrm{~min}$. The covering can prevent the crystals from being corroded over $24 \mathrm{~h}$ in ordinary organic solvents and even stay stable in dichloromethane (DCM) for $120 \mathrm{~h}$.

Organic semiconductor crystals with better properties can be obtained by the solvent evaporation method combined with solvent-resistant coating. In addition, Ye et al. [64] put forward a microspacing in-air sublimation method, in which the device consists of a thermal-stage and two silicon wafers as the bottom and top substrate. The materials are located on the bottom substrate, and the target substrate is covered with the starting material by a small space $150 \mu \mathrm{m}$ away from the bottom substrate, separated by two small glass sleepers. After heating the bottom substrate for a period of time, the microcrystals with good morphology can be obtained.

\section{Side-chain effect}

Morphology modulation can be realized by designing the molecular structure or the side-chain groups, which can adjust the intermolecular interactions and affect the stacking mode during self-assembly. The performance of the optoelectronic devices significantly depend on the morphology of the organic micro/nanocrystals $[37,65,66]$. Wang et al. [67] designed different side chains to modulate the intermolecular interaction for obtaining different organic microcrystals applied to the highefficiency laser optical microresonators [68]. For example, the microstructures based on two $\pi$-conjugated organic molecules were fabricated (Fig. 3a, c) [67]. The 1,4-bis(4-methylstyryl) benzene $(p$-MSB) crystals have a growth morphology of hexagonal microplates, while 1,4-bis(2-methylstyryl)benzene (oMSB) crystals are rectangular microplates (Fig. 3b, d). In order to investigate the influence of the substitutes on the packing mode of the molecule and the photonic property, Yao et al. [69] designed naphthalene derivatives with different intermolecular recognitions as model compounds. The different amino groups for 2-acetyl-6-dimethylaminonaphthalene (AND) and 2-acetyl6-methylaminonaphthalene (AMN) resulted in distinct intermolecular interactions (see insets of Fig. 3e, f). The twodimensional (2D) rhombic nanosheets were caused by the hydrogen-bonding site of amide in $\mathrm{AMN}$, which restrained the packing along asingle direction (Fig. $3 \mathrm{f}, \mathrm{h}$ ). Due to the $\mathrm{H}$ bonding and $\pi-\pi$ stacking interaction, AMN produced a thermodynamically stable sheet-like structure along the [100] and [010] directions. For ADN molecules, a 1D wire-like structure were obtained along the [100] direction because of the $\pi-\pi$ stacking interaction (Fig. 3 e, g). From the above investigations, 

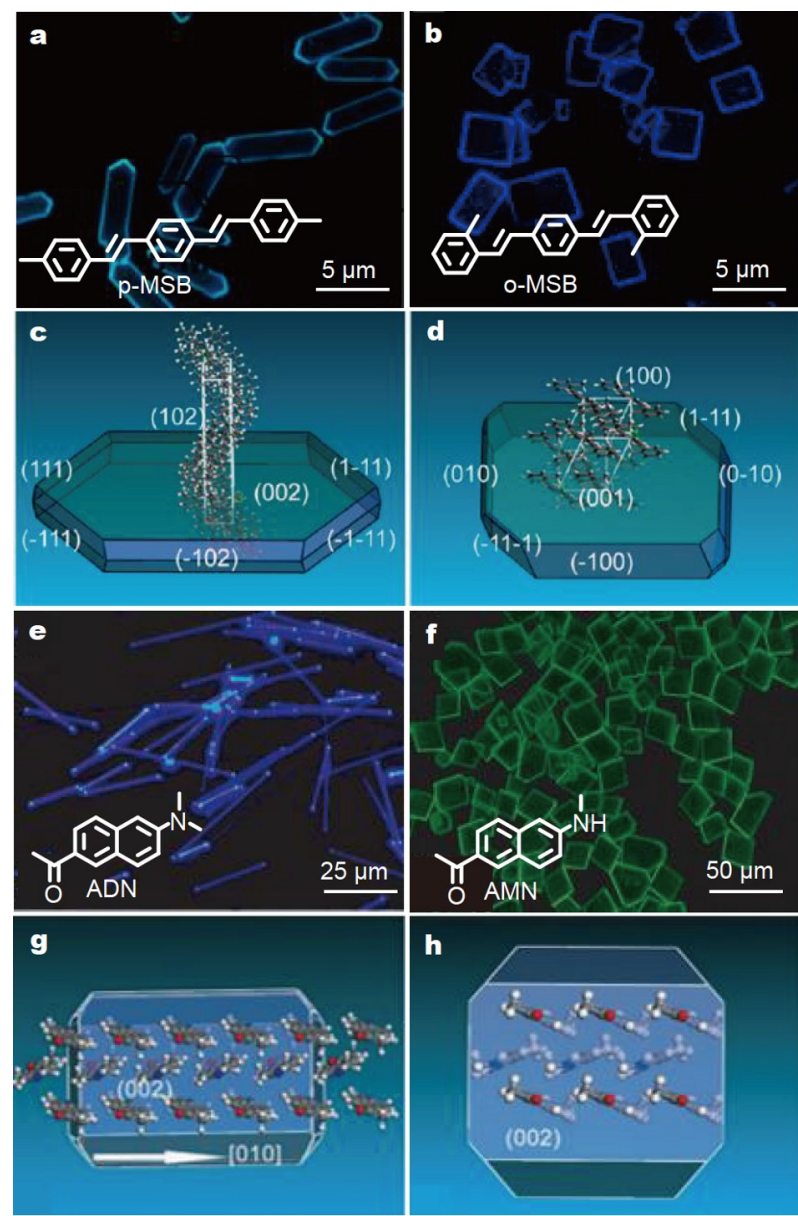

Figure 3 (a, b, e, f) The fluorescence microscopy (FM) images of microcrystals. Insets: the molecular formulas of the model compounds. (c, d, g, h) The predicted growth morphologies of ( $a, b, e, f)$. (a-d) Reproduced with permission from Ref. [67]. Copyright 2014, American Chemical Society. (e-h) Reproduced with permission from Ref. [69]. Copyright 2013, Wiley.

we can find that different side chains on the molecules result in different intermolecular interactions, which are finally responsible for the formation of distinct nanostructures.

\section{Supersaturation degree}

Generally, the change of solubility of organic molecules has a big influence on the supersaturation during nucleation and the subsequent growth kinetics in different planes, which ultimately affect the morphology and crystalline of the organic micro/ nanostructures [70]. Gu et al. [71] obtained 1D rubrene triclinic microwires polymorph at low supersaturation, while $2 \mathrm{D}$ rubrene hexagonal plates were obtained at high supersaturation (Fig. 4a, b). Zhang et al. [72] achieved the controlled synthesis of various high-symmetry morphologies based on 2,5,8,11-tetra-tertbutylperylene (TBPe) crystalline, ranging from cubes to truncated cubes, truncated rhombic dodecahedra, and ultimately to rhombic dodecahedra by the variation of the supersaturation degree of TBPe with the aid of surfactants (Fig. 4c-g). All the morphologies belong to the body-centered cubic structure, in which the particles grow primarily along the [100] or [110] directions (Fig. 4h). The change of supersaturation influences the growth rate of the [100] direction relative to that of the [110] direction. The final nanocrystal morphology depends on the growth rate ratio between the [100] and [110] directions to a large extent (Fig. 4i). The cubes are only covered with the [100] planes, and the rhombic dodecahedra is covered with the [110] planes, of which [110] is the most stable plane in the bodycentered cubic structure.

\section{Solvent- and ultrasound-assisted shape shifting strategy}

Precise control of the morphology with a defined shape and dimension for organic micro/nanostructures is of critical importance due to their potential application in miniature photonic devices such as photonic detectors [73,74], optical waveguides [75] as well as optical sensors [76]. The popular strategy is designing a molecular architecture that includes functional groups, so their anisotropic interactions can generate the desired microphase separation and local packing [77-79]. However, it is still a challenge to prepare shape-shifting micro/ nanostructures in organic-based nanoscience [80]. A few studies on reversible shape-shifting induced by external conditions have been reported. For example, Ajayaghosh et al. [81] have realized a spherical to helical transition for tripodal squaraines by a cation-induced process. Chandrasekar and his colleagues [82] reported a novel bottom-up method to fabricate $1 \mathrm{D}$ nanorings, 1D nanotubes, and 2D nanosheets by controlling the factors of solvents, ultrasound, and time to fabricate organic optical waveguides with reversible shape-shifting. Impressively, the change in shape between $2 \mathrm{D}$ nanosheets and $1 \mathrm{D}$ nanotubes is reversible. In this experiment, the 1,4 -bis $\left(1,2^{\prime}: 6^{\prime}, 1^{\prime \prime}\right.$-bis(3-butyl$1 H$-3,4,5-triazolyl)pyridin-4'-yl)benzene (1, Fig. 5a) was selfassembled to form rhombus-shaped nanosheets in $\mathrm{CH}_{3} \mathrm{CN}$ (Fig. 5b). In the presence of water, the nanosheets were transformed to nanotubes by a rolling mechanism (Fig. 5c). After the ultrasonic treatment, the nanotubes were transformed back to nanosheets. Interestingly, nanorings were formed through the bending and coiling mechanism from the nanotubes after being immersed in the solution for one week (Fig. 5d). Thus, both solvent and ultrasonic can induce the mechanical deformation of organic solids at appropriate time intervals to obtain organic solids with three different dimensions such as $2 \mathrm{D}, 1 \mathrm{D}$, and $0 \mathrm{D}$. The detailed studies on the mechanism of shape-shifting show that the fundamental driving force of the plastic deformation of the solids is the hydrophobic surface energy.

\section{Solvent etching}

The preparation of morphology-shifting micro/nanocrystals is becoming one of the most cutting-edge research areas in organic nanoscience, because the functions of the organic micro/nanocrystals rely on their sizes and dimensions [83]. The solvent etching approach, firstly proposed by Chandrasekar's group [82], is a very convenient and commonly used method for the fabrication of organic crystals, and plays an important role in the shape-shifting of organic micro/nanostructures. This approach depends on the huge difference in the solubility of molecules in good and poor solvents. The micro-environment has been changed when the stock solution is exposed to a poor solvent, which influences the nucleation and growth process of the micro/nanocrystals. Several groups have obtained organic micro/nanostructures with regular morphologies by the solvent etching method. For example, Wang and his colleagues [84] have demonstrated a synergistic bottom-up and top-down approach to obtain organic single crystalline nanowires (OSCNWs) by this method. As shown in Fig. 6a, the (E)-3-(4- 
a

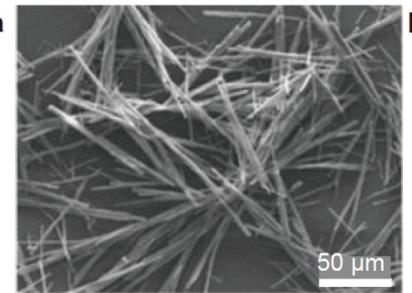

d

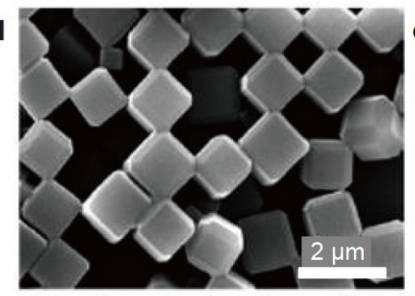

g

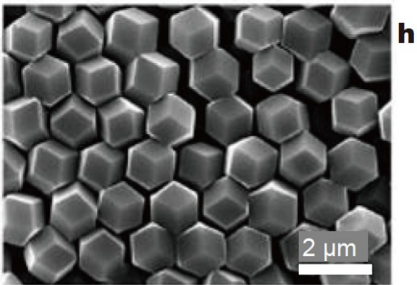

h
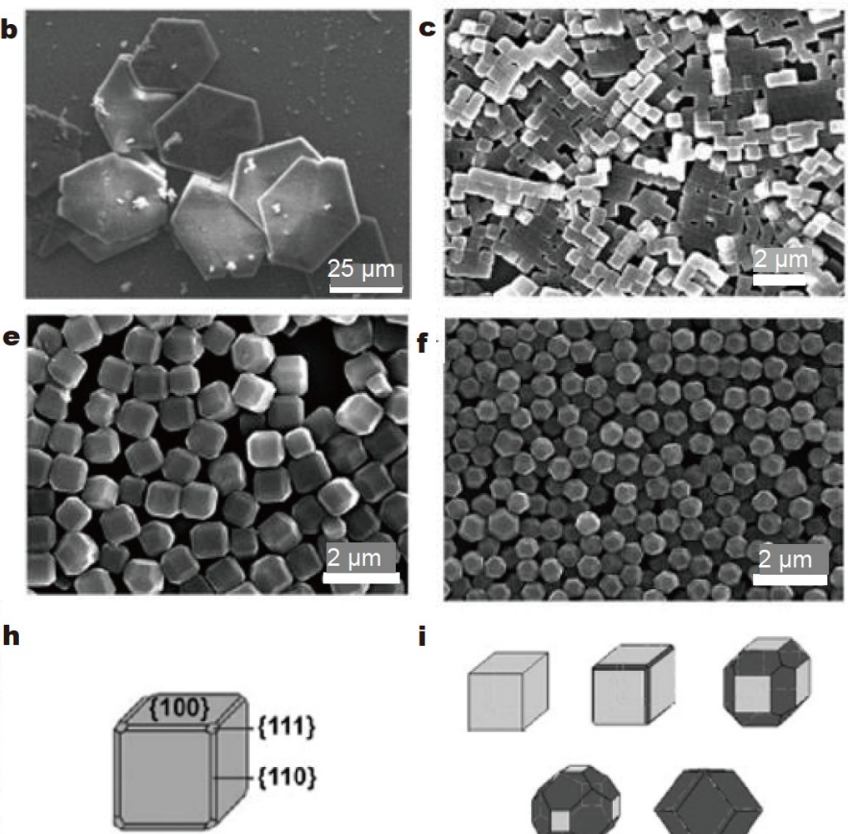
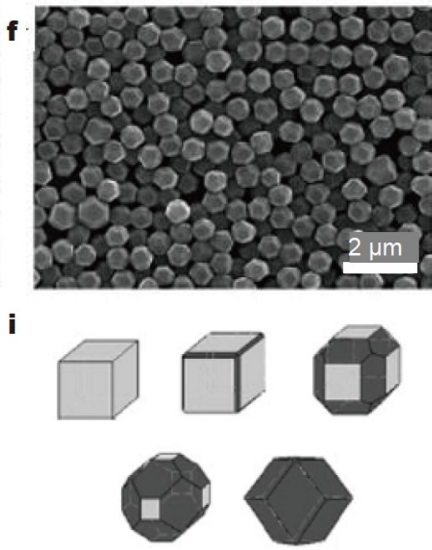

Figure 4 Scanning electron microscopy (SEM) images of (a) 1D microwires polymorph and (b) 2D hexagonal plates. Reproduced with permission from Ref. [71]. Copyright 2017, American Chemical Society. SEM images of the as-prepared particles: (c) cubes; (d) truncated cubes; (e) intermediate shape; (f) truncated rhombic dodecahedra; (g) rhombic dodecahedron. (h) Unit cell of the cubic lattice. (i) Geometrical particle shapes in the [100] and [110] directions. Reproduced with permission from Ref. [72]. Copyright 2009, Wiley.

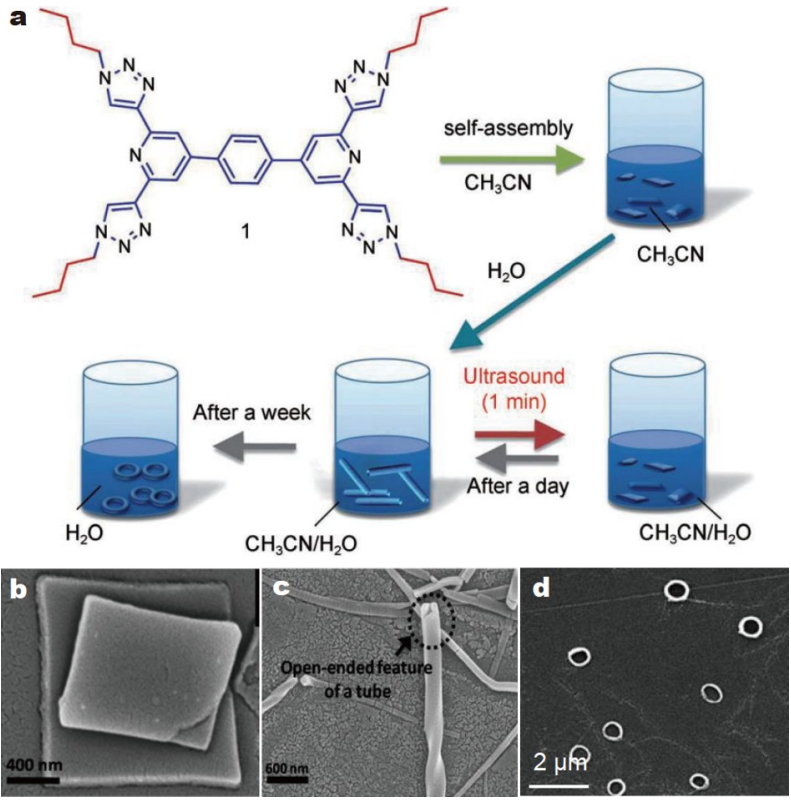

Figure 5 (a) The chemical formula of molecular 1 and the schematic illumination of the shape-shifts under different self-assembly conditions. The SEM images of the (b) nanosheets, (c) nanotubes, and (d) nanorings. Reproduced with permission from Ref. [82]. Copyright 2012, Wiley.

(dimethylamino)-2-methoxyphenyl)-1-(1-hydroxynaphthalen2-yl)prop-2-en-1-one (DMHP) solution in DCM was poured into a poor solvent of ethanol. Taking advantage of the solvent etching process motivated by ethanol, organic microrods synthesized by the bottom-up method transform into organic microrods with small holes. Subsequently, the microrods with

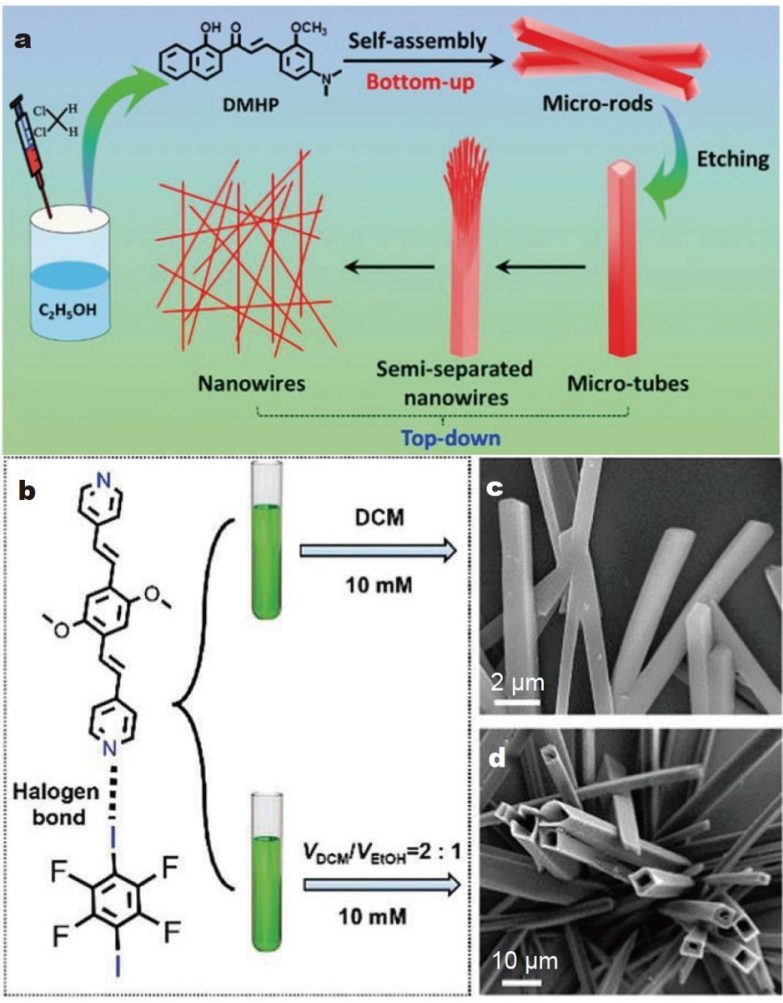

Figure 6 (a) Schematic illumination of the morphological evolution of DMHP. Reproduced with permission from Ref. [84]. Copyright 2018, Royal Society of Chemistry. (b) Schematic illumination of the fabrication of the DPEpe- $\mathrm{F}_{4} \mathrm{DIB}$ microcrystals. (c, d) SEM images for the obtained microcrystals. Reproduced with permission from Ref. [85]. Copyright 2018, Royal Society of Chemistry. 
small holes can be further transformed to organic microtubes with the sufficient etching process, which affords a top-down method for the preparation of the final nanowires. This solvent etching approach can apply to the preparation of not only single-component organic micro/nanostructures, but also binary organic single-crystal microrods and microtubes. In our another study, 4,40-((1E,10E)-(2,5-dimethoxy-1,4-phenylene)bis(ethene2,1-diyl))dipyridine (DPEpe) and 1,4-diiodotetrafluorobenzene $\left(\mathrm{F}_{4} \mathrm{DIB}\right)$ single-crystals were obtained via modulating the solvent system, which exhibited microrods and microtubes morphology (Fig. 6b) [85]. When DPEpe and $\mathrm{F}_{4} \mathrm{DIB}$ were mixed in DCM, microrods were generated after all the solvent evaporated (Fig. 6c). By comparison, the microtubes were obtained when a mixture solvent of DCM and ethanol was used to dissolve DPEpe and $\mathrm{F}_{4} \mathrm{DIB}$ under the same conditions (Fig. 6d). This simple solvent etching approach is conducive to the controlled manufacture of OSCNWs, which may be the building-blocks for the next-generation miniaturized optoelectronic devices on the micro/nano scale.

\section{Vapor deposition}

Vapor deposition (VD) is widely applied to the preparation of inorganic nanomaterials and great success has been achieved in fabrication of $1 \mathrm{D}$ inorganic nanostructures [86,87]. However, in the preparation of organic nanocrystals, the monodispersity of the target product is hard to regulate by the VD method [88-91]. Jiang and his colleagues [92] guided the small molecules to nucleate and grow by utilizing Si pillar substrates, and obtained the organic micro/nanocrystals (Fig. 7a). Different from the traditional VD technology, this allows organic semiconductors to be orderly dispersed on the substrate, solving the problem of disordered dispersion. The molecules prefer to nucleate at the edges of the substrate because of the energy differece of the top and side substrate surfaces (Fig. 7b). Consequently, the desired morphologies can be achieved by joining these nanowires together at different angles through changing the geometries of the substrates. As shown in Fig. 7c, 9,10-bis (phenylethynyl) anthracene (BPEA) molecules nucleated and grew along the pentagonal pillar edges. As a result, the organic wires were bonded at the corner of the micropillar substrates, and ultimately formed the organic semiconductor microcrystals. When focusing the laser beam on this structure, the PL arrange structure was largely guided by rules. Except the linear patterns, connected BPEA lines can produce pentagons, hexagons (Fig. 7d), or even circles (Fig. 7e), by joining nanowires together at different angles via the PVD strategy, which possess good photoelectric properties and stability.

\section{Template-assisted route}

The template-assisted approach is also used as a regular method to synthesize 1D inorganic nanostructures. Possin [93] was the first to utilize an insulating matrix to produce metal wires of very small diameters. Martin et al. [94,95] first defined this method as "template synthesis" and extended it for the fabrication of conductive polymer nanostructures. Nowadays, the most frequently used templates include ordered porous membranes prepared from anodized alumina (AAO) [96], nanochannel glass [97], and silica [98] in the template-assisted method. In recent years, the template-assisted method has aroused wide concern in the preparation of organic structures

a

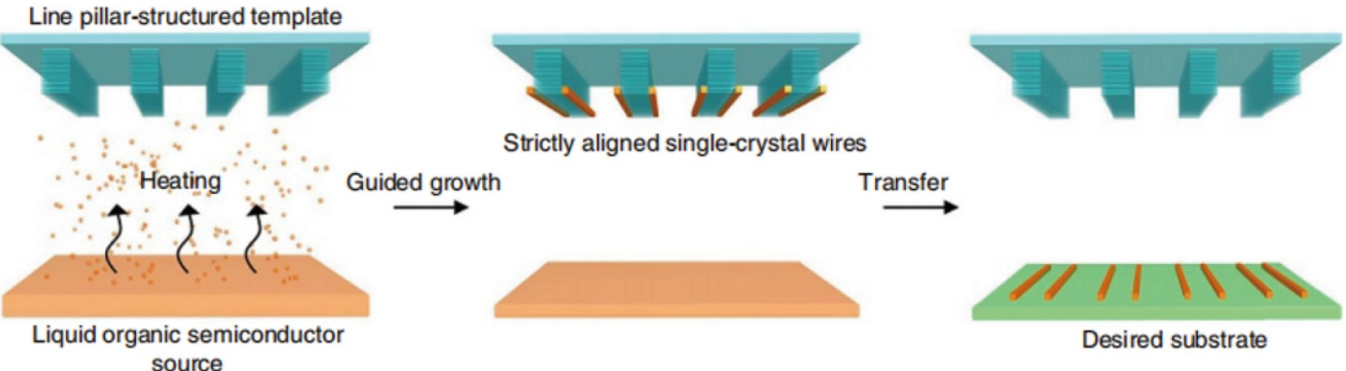

b
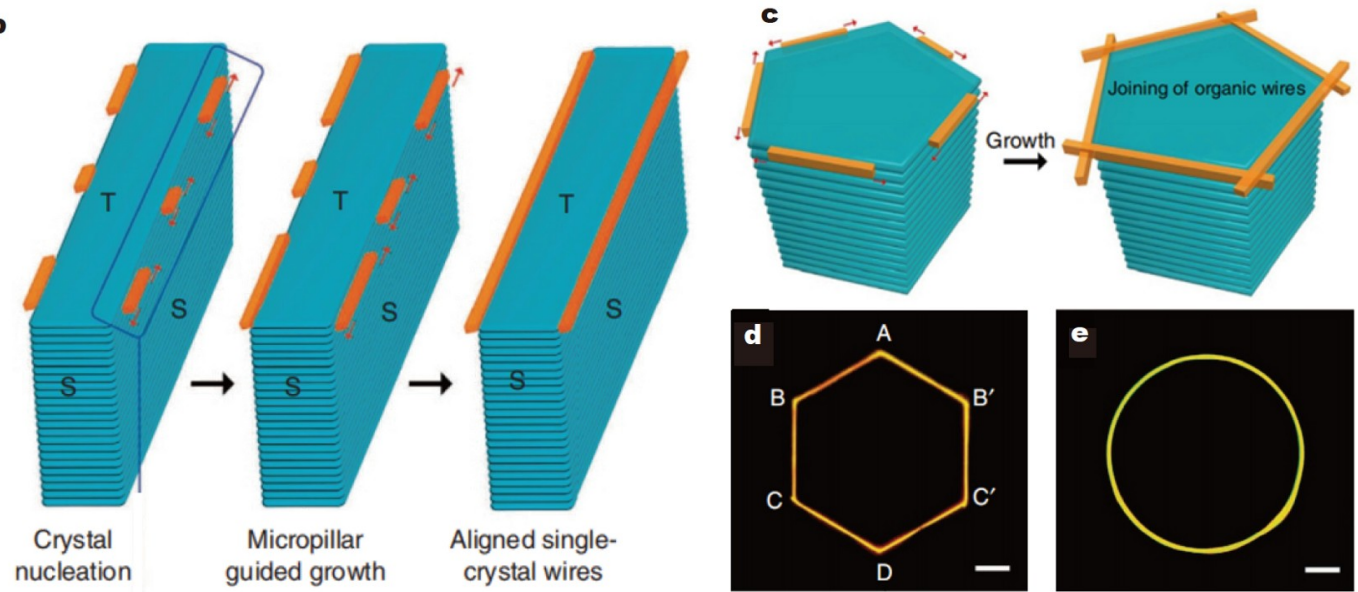

Figure 7 (a) The schematic diagram for surface-induced growth of 1D organic crystals. (b) Schematic illustration of the crystal nucleation and growth of BPEA wires. (c) The schematic diagram of joined BPEA crystal. Dark-field FM image of hexagonal (d) and circular (e) wires. Reproduced with permission from Ref. [92]. Copyright 2015, Macmillan Publishers Limited. 
based on organic small molecules. For example, Yao and coworkers [99] realized the large-area synthesis of an organic nanonet structure with the help of an AAO template. The organic nanonet composed of uniform 5,10,15,20-tetrakis( $p$ chlorophenyl)porphyrin (TClPP) nanoparticles was fabricated floating on the AAO template (Fig. 8a). When the AAO template was removed, the weblike nanonet structure of TCIPP was remained well and the pore size mimicked that of the AAO template (Fig. 8b). Obviously, the ordered holes of AAO template contributed to forming the periodic structure of the nanonet on the reverse of the template. The AAO templates with hole sizes of 50, 100 and $200 \mathrm{~nm}$ were used in the synthesis of TCIPP nanonets as shown in Fig. 8c-e. Although stable nanonets of TCIPP can be reproduced by this method, the extension of this strategy to other molecules still needs to be explored.

\section{Adsorbate/surfactant-assisted route}

The adsorbate/surfactant-assisted route is also called the soft template method, including surfactant micelles [100], small adsorbates [101], copolymers [102], biomolecules [103], and complexes [104]. This method induces the target molecules to assemble according to the pattern of the template. It is well known that as the concentration of surfactant solution reaches the critical micelle concentration, micelles with different morphologies will appear. Kang et al. [105] prepared perylene nanoparticles (PeNPs) using cetyltrimethylammonium bromide $\left(\mathrm{CTA}^{+} \mathrm{Br}^{-}\right)$as the surfactant by a colloid chemical reaction method, where the sizes of PeNPs can be adjusted by the variation of the monomer concentration and the injection methods. Through altering the molar ratio $(N)$ of reductant $\mathrm{CTA}^{+} \mathrm{Br}^{-}$and $\mathrm{Pe}^{+} \mathrm{ClO}^{4-}$, the size of PeNPs can be modulated easily, due to

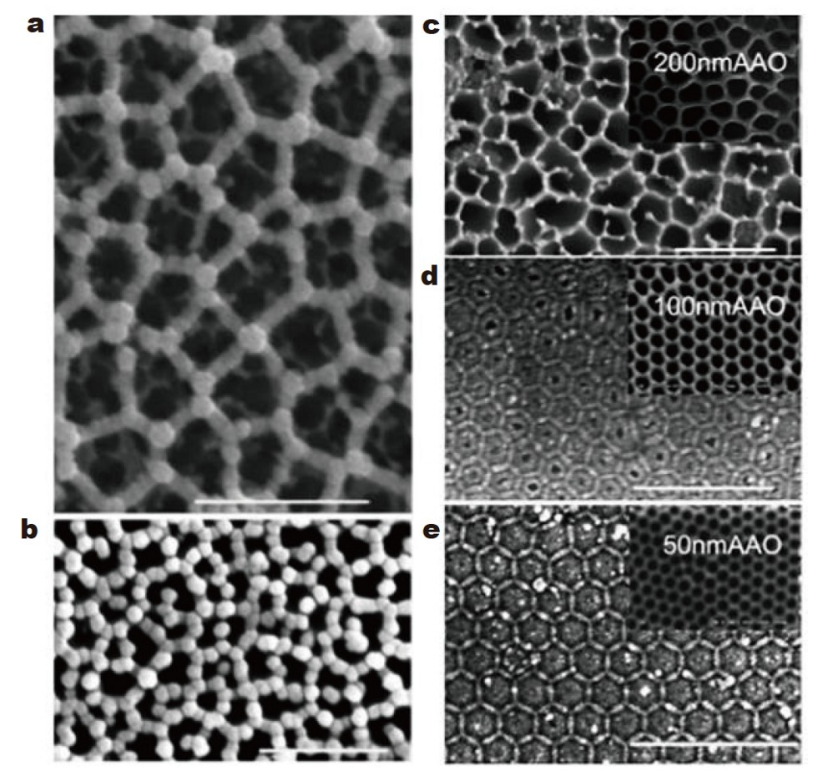

Figure 8 SEM images of TClPP nanonets. (a) A floating nanonet. (b) A freestanding TCIPP nanonet after the AAO template is removed. (c-e) TClPP films in line with the pore sizes of the AAO templates. Scale bars: $500 \mathrm{~nm}$. Reproduced with permission from Ref. [99]. Copyright 2008, Wiley.

different amounts of perylene molecules generated. Fig. 9a shows that when $N<0.2$, particles cannot be formed. Nanoparticles can be formed when the value of $N$ is between 0.2 and 0.3 . When the value of $N$ is between 0.3 and 1.0, the size of the formed particles will further increase. As shown in Fig. 9b-d,

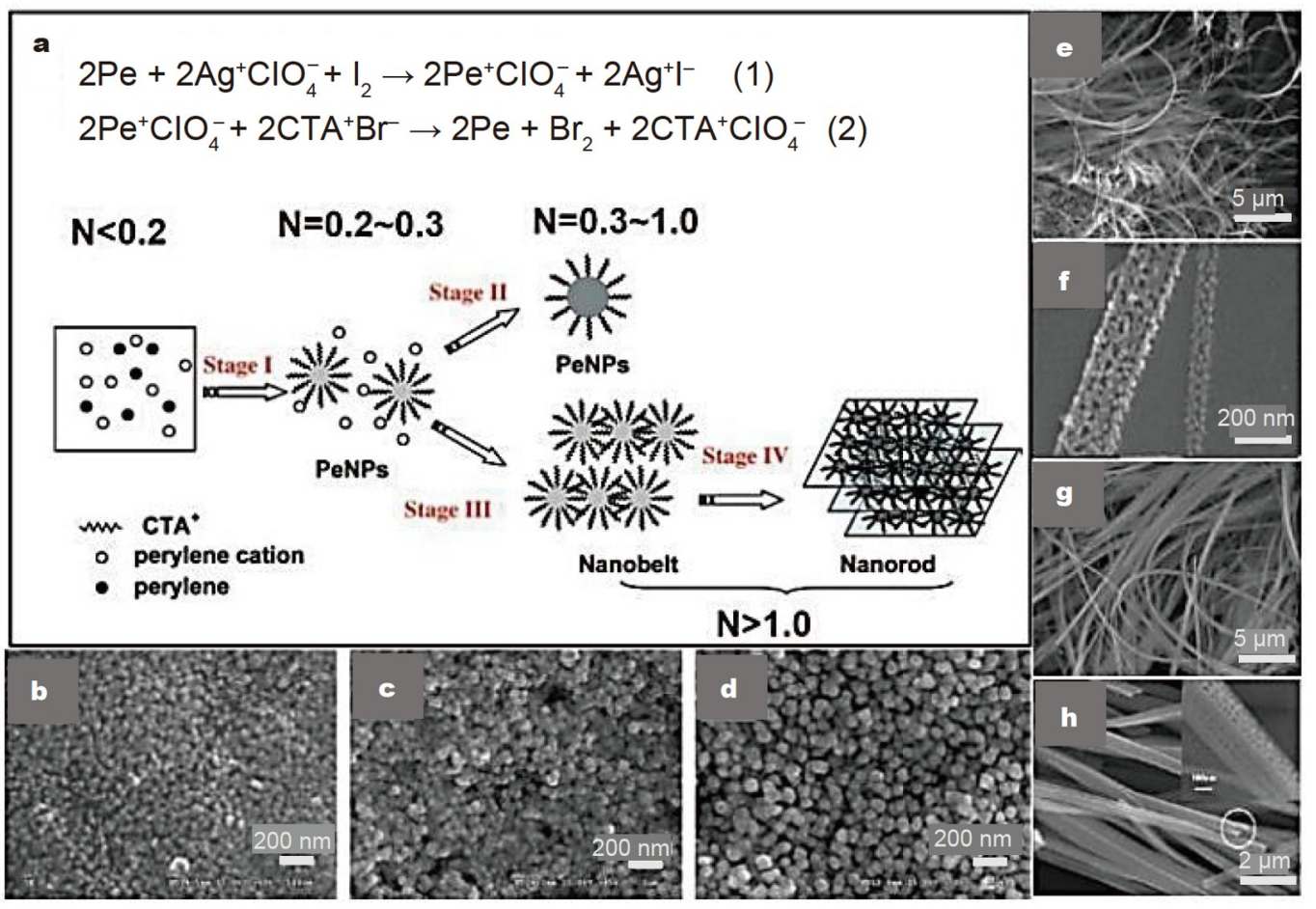

Figure 9 (a) The reaction equation for the formation of Pe and the schematic illustration of the formation of quasi-spherical PeNPs. (b-d) SEM images of PeNPs with sizes of (b) $\sim 25 \mathrm{~nm}$ at $N=0.3$, (c) $\sim 60 \mathrm{~nm}$ at $N=0.6$ prepared by the droplet injection, and (d) $\sim 90 \mathrm{~nm}$ at $N=1.0$ prepared by the singlet injection. (e) $N=1.2$. (f) $N=1.0-1.2$; intermediate states. (g) $N=1.4$; nanobelts. (h) $N=2.0$; square nanorods. Reproduced with permission from Ref. [105]. Copyright 2007, American Chemical Society. 
large-scale nearly monodisperse nanoparticles with the size ranging from 25 to $90 \mathrm{~nm}$ can be prepared along with increasing $N$. In addition, they also found that the injection method such as singlet or droplet method can affect the sizes of the PeNPs even at the same $N$ value. Under the droplet injection method and $N$ $>1.0$, the obtained PeNPs possess almost the same size to that obtained at $N=1.0$. In contrast, by using the singlet injection method at $N>1.0$, belt-like structures arranged row by row can be obtained which are composed of PeNPs of $25 \mathrm{~nm}$ (Fig. 9e), while at $1.0<N<1.2$, the nanowires can be observed in Fig. 9f out of a large amount of PeNPs and nanosheets formed with tight attachment of nanowires. As the value of $N$ increases to 1.4, thick nanobelts are produced (Fig. 9g). Further increasing the value of $N \geq 2.0$, nanorods with a square shape are obtained, which are assembled under a layer-by-layer self-assembly mechanism (Fig. 9h). High resolution SEM (HRSEM) images exhibit that the nanorods are composed of $25 \mathrm{~nm}$ PeNPs (inset of Fig. 9h). The above experimental results indicate that the $25 \mathrm{~nm}$ PeNPs are the basis of nanowires, nanosheets, and nanorods. With the assistance of surfactants, nanoparticles with different sizes and highly ordered organic nanomaterials can be obtained by this colloidal chemical reaction method.

\section{OPTICAL PROPERTY MODULATION}

Besides the morphology modulation, much attention is focusing on modulating the optical performance and the investigation of the photo-physical process for the organic micro/nanocrystals. Different from the Wannier excitons in inorganic semiconductors [106], the optoelectronic properties for the organic materials depend on the CT excitons and Frenkel excitons with smaller radii [107,108]. Tao et al. [109] prepared organic microcrystals with switchable fluorescence and phosphorescence by forming halogen bond between the host and guest molecules in the crystal matrix of host material. Beyond that, Wang and his colleagues [110] explored the shape-dependent microresonator properties based on 3-[4-(dimethylamino)phenyl]-1-(2-hydroxyphenyl)prop-2-en-1-one (HDMAC). They successfully prepared microcrystals with shape-dependent microresonator characteristics, in which the 1D microwires function as a FabryPérot-mode optical resonator and 2D microdisks act as a WGM optical resonator. Thus, the photoelectric properties of organic materials and inorganic materials are substantially different. In this section, the modulation approach of the optical properties for organic micro/nanocrystals has been discussed.

\section{Förster resonance energy transfer (FRET) routes}

The FRET process has been widely investigated for the construction of optical devices such as OLED and tuning the emission color [111-113]. In an FRET process, when stimulated by light, excitons occupying on the $S_{1}$ level formed instantly by the donor organic molecules. The exciton energy of donor molecules then efficiently transfers to $S_{1}$ energy level of the acceptor molecule via the FRET process, which ultimately emits the corresponding light from the acceptor molecules. One approach to realize FRET is the doping technique, which will efficiently enhance the luminous efficiency and the luminous color for organic materials. Efficient energy transfer via FRET is identified to be responsible for the enhanced emission from the acceptor. Li et al. [114] demonstrated the fabrication of multicolor organic microcrystals taking advantage of FRET, in which $o$-MSB was chosen as the host molecules and tetracene or DBP were chosen as the guest molecules (Fig. 10a). As shown in Fig. $10 \mathrm{a}$, the absorbed energy of the $o$-MSB can be transferred to tetracene or DBP, and then released via light emission, greatly preventing the reabsorption by the organic molecules. The FM image of the $o$-MSB microplates exhibits intensive sky blue light emission (Fig. 10b). Meanwhile, the tetracene-doped $o$-MSB shows strong green light emission and the DBP-doped $o$-MSB microcrystal exhibits the orange light emission, which is ascribed to the FRET process from $o$-MSB to tetracene/DBP molecules (Fig. 10c, d). In addition, the doping of the acceptor organic molecules has no effect on the shape of the as-prepared organic micro/nanostructure. From the fluorescence spectra, it can be seen that the maxima at 450, 500 and $550 \mathrm{~nm}$ for the $o$-MSB, tetracene-doped $o$-MSB, and DBP-doped $o$-MSB microcrystals, respectively (Fig. 10e). The emission band of $450 \mathrm{~nm}$ for $o$-MSB microcrystals was still observed in the doped microcrystals, which indicates an incomplete FRET process. The calculated energy transfer efficiency of the tetracene-doped $o$-MSB microcrystal is $44.3 \%$, while that of the DBP-doped $o$-MSB microcrystal is $67.4 \%$. In addition, the emission light color can be modulated from blue to yellow through controlling the doping ratio (Fig. 10f1-f6). As the doping ratio of DBP is 1.5:100, the organic microcrystals exhibit bright white light emission (Fig. 10f4). As we all know, the white emissive organic microcrystals have widespread applications in integrated optoelectronic devices.

\section{Chemical reactions}

Chemical reaction has been widely utilized to synthesize organic micro/nanostructures in the solution phase where the reactant precursors possess good solubility and the target product can be

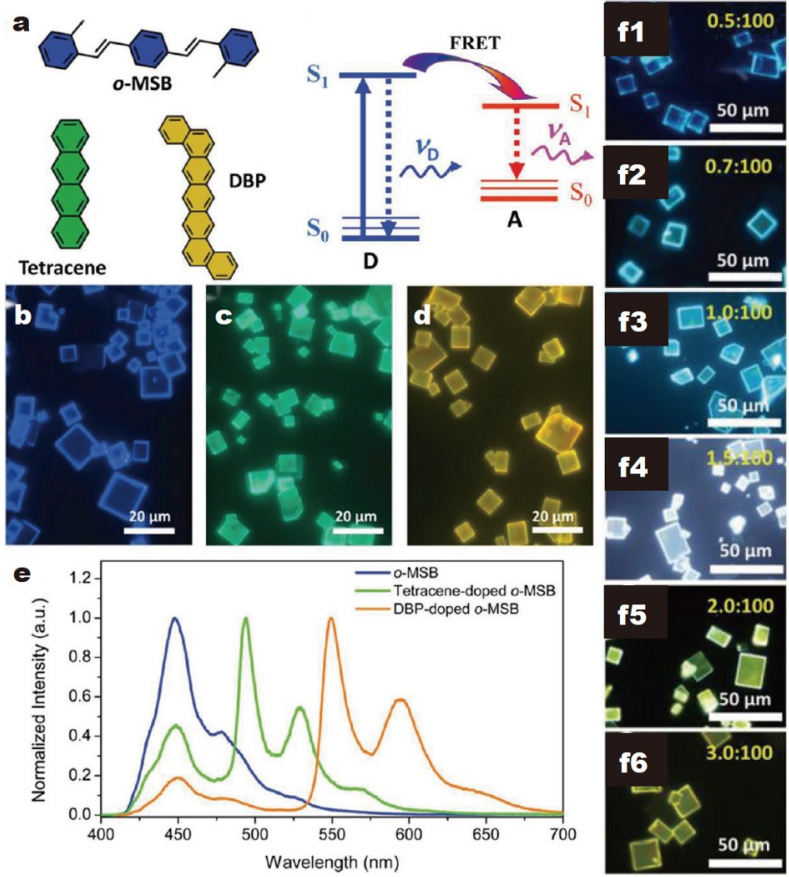

Figure 10 (a) The molecular structures and the schematic diagram of the FRET process from a donor to an acceptor. (b-d) FM images of the obtained microcrystals. (e) PL spectra of doped $o$-MSB microcrystals. (f1-f6) The FM images of microcrystals with different doping ratios. Reproduced with permission from Ref. [114]. Copyright 2008, Wiley. 

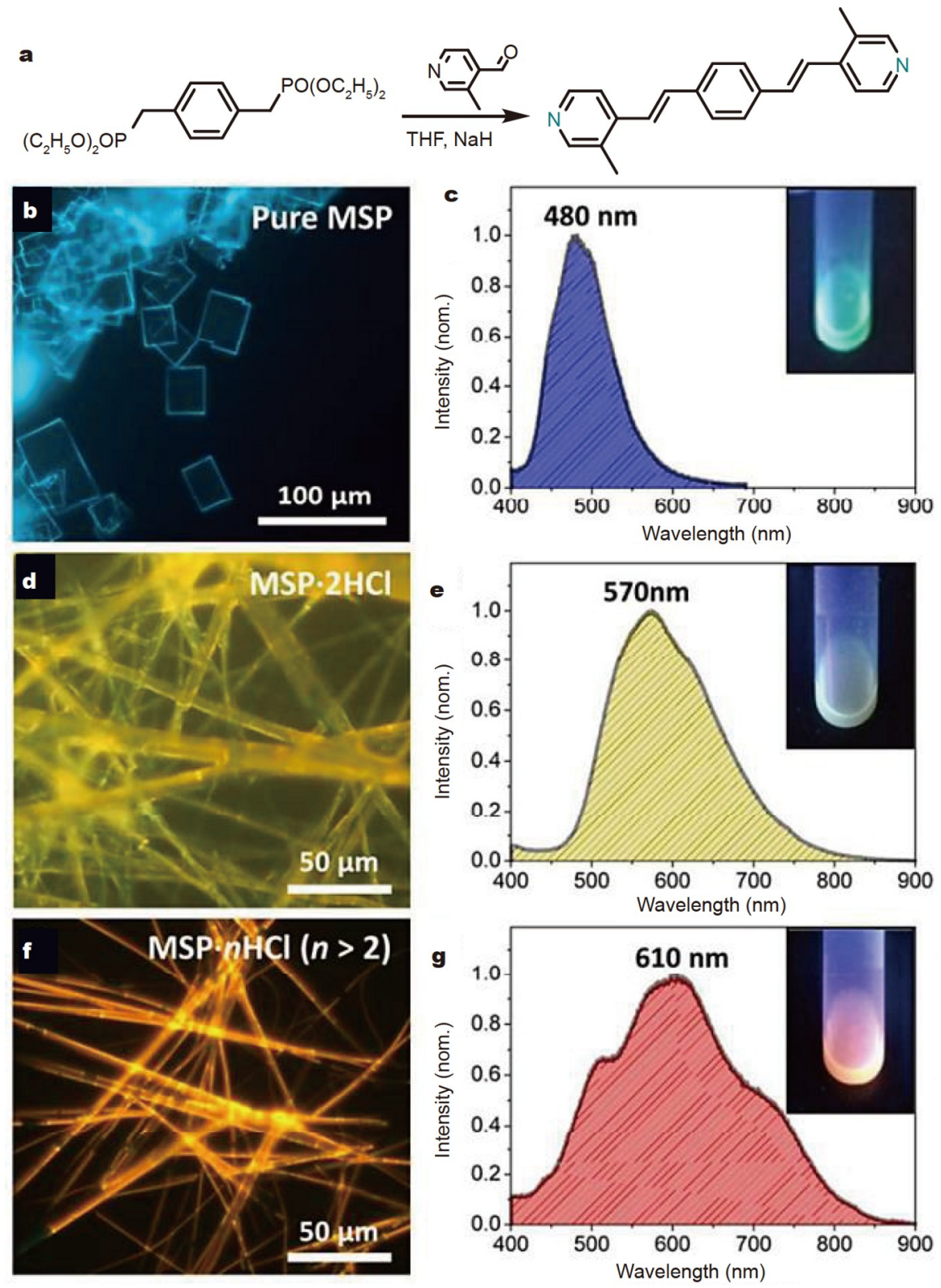

Figure 11 (a) The synthesis route of MSP. (b, d, f) FM images of the as-prepared microcrystals. (c, e, g) The PL spectra for the microcrystals. Insets: photographs of the as-prepared products dispersed in ethanol. Reproduced with permission from Ref. [120]. Copyright 2017, Royal Society of Chemistry.

formed as a precipitate. The homogeneous solution phase can continuously adjust the supersaturation of the target product during precipitation, which renders it possible to precisely modulate the complex nucleation process initially and the subsequent fast growth $[115,116]$. Compared with the reprecipitation method, this method can obtain organic nanoparticles with regular morphology and narrow size distribution, which avoid becoming the building-blocks for further self-assembly $[117,118]$. On the other hand, the chemical reaction method makes the large-scale synthesis of organic nanoparticles possible. Consequently, the chemical reaction route can be used to modulate the morphology of the organic micro/nanostructure, finally tuning the optical properties [119].

Recently, Wang and his colleagues [120] also realized the luminescence-/morphology-tunable organic microcrystals using the protonation reaction (Fig. 11a). As shown in Fig. 11b, the pure 2D plate-like structure 1,4-bis((E)-2-(3-methylpyridin-4-yl) vinyl)benzene (MSP) microcrystals exhibit bright blue emission light at the four edges of the rectangular structure but a faint emission from the bodies, exhibiting $2 \mathrm{D}$ optical confinement. The central emission for pure MSP is located at $480 \mathrm{~nm}$ (Fig. 11c). The molar ratio of MSP to $\mathrm{HCl}$ was controlled to be 1:2 when adding $\mathrm{HCl}$ to the MSP solution, and $\mathrm{MSP} \cdot 2 \mathrm{HCl}$ microwires were obtained, which exhibit yellow-emission light corresponding to a maximal emission wavelength at $570 \mathrm{~nm}$ (Fig. 11d, e). From the above results, it can be seen that the protonation has taken place and the fluorescence and morphology have also been modulated. The $\mathrm{MSP} \cdot n \mathrm{HCl}$ microcrystals were obtained with a microwire morphology and red light emission, while excess amount of $\mathrm{HCl}$ was added to the MSP solution (Fig. 11f). The fluorescence spectrum maximum is located at $610 \mathrm{~nm}$ (Fig. 11g), exhibiting obvious red-shift com- 
a

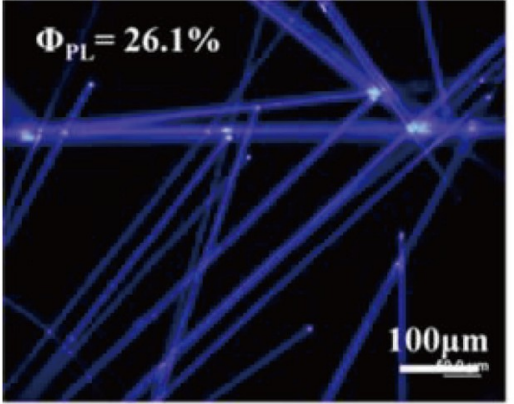

c

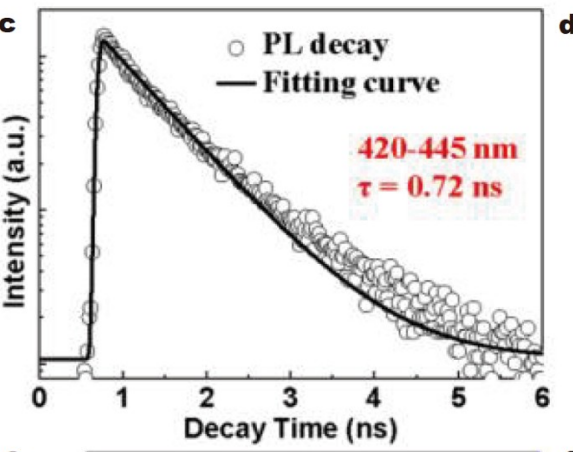

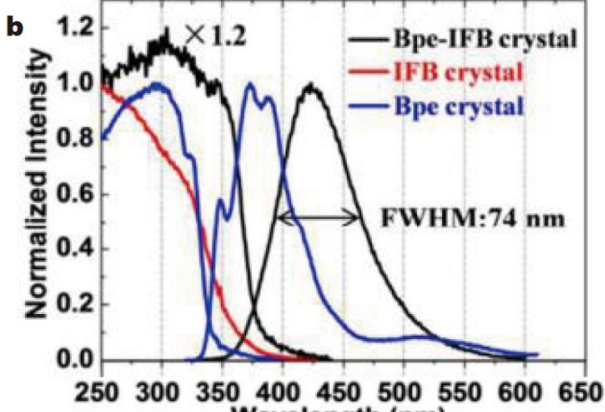

Wavelength (nm)

d

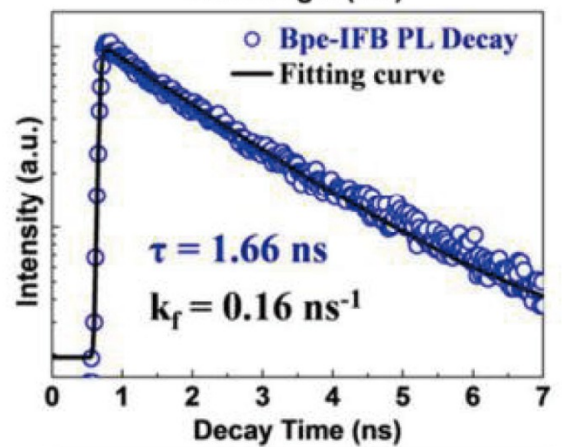

e
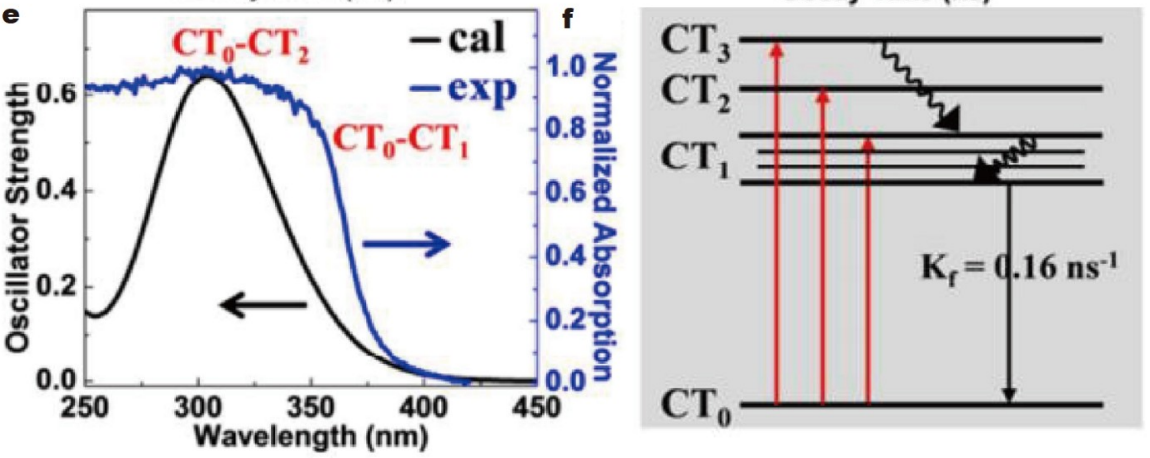

Figure 12 (a) CLSM images of BICs. (b) The PL and absorption spectra of Bpe, IFB, and Bpe-IFB crystals. Time-resolved PL measurements of (c) Bpe and (d) BICs. (e) The absorption spectra calculated based on TD-DFT (black curve) and experimental result (blue curve) of BICs. (f) The Jablonski diagram of BICs. Reproduced with permission from Ref. [122]. Copyright 2015, American Chemical Society.

pared with that of the pure MSB microcrystals. However, for MSP. $n \mathrm{HCl}(n>2)$ microcrystals, the $n$ value could not be exactly determined, due to the formation of ionized crystals when $\mathrm{HCl}$ is added. Similar phenomenon has also been observed in pyridinehydrogen fluoride system and pyridine-formic system reported by Mootz's group [121]. In our reported work, MSP also protonates with other acids such as sulfuric acid.

\section{Excited state modulation}

Furthermore, Zhu et al. [122] selected 1,2-di(4-pyridyl)ethylene (trans-Bpe) and 1,3,5-trifluoro-2,4,6-triiodobenzene (IFB) as donor/acceptor (D/A) molecules to obtain Bpe-IFB co-crystals (BICs) via a crystal engineering strategy. Fig. 12a exhibits the confocal laser scanning microscope (CLSM) images of BICs, which shows strong blue luminescence at the tip or edge, and the PLQY $\left(\varphi_{\mathrm{PL}}\right)$ is measured to be $26.14 \%$. What's more, the PL and absorption spectra of BICs are collected (Fig. 12b). At $425 \mathrm{~nm}$, there is a red shift PL and a full width at half maximum (FWHM) about $74 \mathrm{~nm}$, which implies it maybe rise in CT transitions. Time-resolved PL measurements further clarify the nature of this amusing phenomenon and render a deeper understanding on the excited states of co-crystals. The PL of cocrystals reaches its peak at $425 \mathrm{~nm}$ and the lifetime $(\tau)$ is about $1.66 \mathrm{~ns}$, which is quite different from the PL of Bpe crystals (Fig. 12c, d), indicating a new PL excited species was generated in the co-crystals. The radiation rate $\left(k_{\mathrm{f}}\right)$ of BICs $\left(0.16 \mathrm{~ns}^{-1}\right)$ is smaller than that of the single-component crystals calculated by the formula of $k_{\mathrm{f}}=\Phi_{\mathrm{PL}} / \tau$, implying its CT transition nature. As shown in Fig. 12e, through time-dependent density functional theory (TD-DFT) calculated results, the absorption spectra of BICs and electronic transitions are marked.

According to the experimental and theoretical evidences, researchers draw the Jablonski diagrams of BICs (Fig. 12f). Within BICs, when the photons are absorbed, the electron will transition from $\mathrm{CT}_{0}$ ground state to $\mathrm{CT}_{n}$ excited state, then relaxes to the lowest $\mathrm{CT}_{1}$ state and fluoresces through large molecular configuration changes.

\section{Shape-dependent optical properties}

The shape and dimensions of the organic micro/nanostructures have great impact on the guidance of optical waves and the light confinement, and thus the construction of diverse structures is 

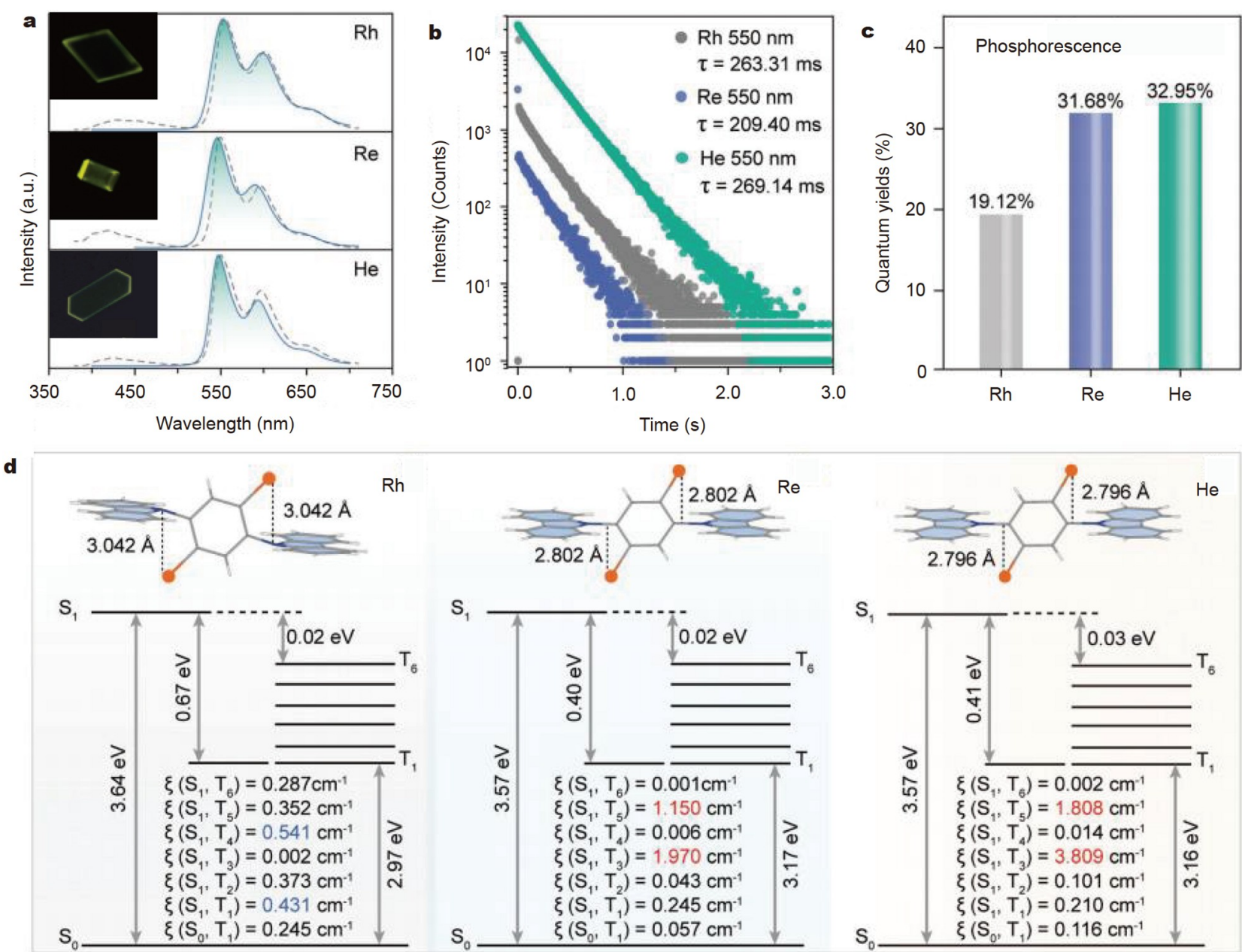

$\mathrm{S}_{1}$

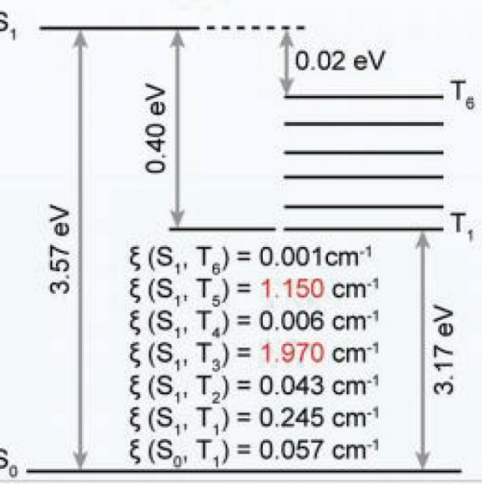

$\mathrm{S}$,

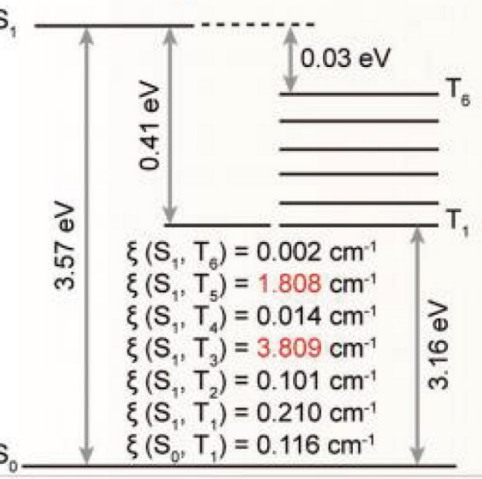

Figure 13 (a) Phosphorescence (solid lines) and steady-state PL (dash lines) spectra of Rh, Re and He, respectively. Insets: the corresponding FM images of $\mathrm{Rh}, \mathrm{Re}$ and He. (b) Lifetime distributions of phosphorescence emission bands at $550 \mathrm{~nm}$ and (c) the phosphorescence efficiencies of Rh, Re and He, respectively. (d) The calculated SOC constants, energy diagrams and the selected SOC $(x)$ models of Rh, Re and He, respectively. Reproduced with permission from Ref. [126]. Copyright 2021, Royal Society of Chemistry.

essential for controlling the photonic flow [123]. For example, the optical waveguide can only be observed in high-quality lowdimensional organic materials with the field confinement in two dimensions [124]. Otherwise, the 2D sheet-like structure confining photons to one dimension can be integrated into a chipscale planar photonic system [125].

Recently, Huang and his colleagues [126] explored the shapedependent optical properties based on one ultralong organic phosphorescence compound 9,9'-(2,5-dibromo-1,4-phenylene) bis $(9 H$-carbazole) (PDBCz). In their experiment, three types of 2D microstructures (rectangular (Rh), rectangular (Re) and hexagonal $(\mathrm{He})$ ) were obtained by controlling the concentration of solution via a self-assembly strategy. Rh crystals were successfully induced at low molecular concentration $\left(1 \mathrm{mmol} \mathrm{L}^{-1}\right)$; when the molecular concentration reached 5 and $8 \mathrm{mmol} \mathrm{L}^{-1}$, Re and $\mathrm{He}$ crystals were obtained, respectively (see the inset of Fig. 13a). On account of the variation of molecular stacking patterns, the photophysical properties of the crystals depend sensitively on their morphology. The relationship between the photophysical properties and the structure of crystals was further studied. The authors measured the steady-state PL and phosphorescence spectra of these microstructures under ambi- ent conditions. The PL spectra of the three crystals are dominant at $550 \mathrm{~nm}$ with bright-yellow emission (Fig. 13a), and the ultralong luminescence lifetimes of the Rh, Re and He crystals are 263.31, 209.4 and $269.14 \mathrm{~ms}$, respectively (Fig. 13b). What's more, the phosphorescence efficiency of Rh crystals $(19.12 \%)$ is lower than $31.68 \%$ and $32.95 \%$ for Re and He crystals respectively, (Fig. 13c). The authors used theoretical calculations to reveal why these crystals possess different phosphorescence efficiencies. They believe that disparate molecular conformations lead to different intramolecular/intermolecular heavy atomic effects, resulting in different spin-orbit coupling (SOC) constants $(x)$ between $S_{1}$ and $T_{n}$, but this effect has little influence on the rate constant of the intersystem crossing (ISC) process of $\mathrm{Rh}$ (Fig. 13d). Consequently, the phosphorescence efficiency of Rh is lower than those of Re and He. It is rational to speculate that the molecular conformations of different crystal types can effectively affect the phosphorescence properties.

Another study focusing on the shape-dependent optical modulation is based on binary organic 4,40-((1E,10E)-(2,5dimethoxy-1,4-phenylene)bis(ethene-2,1-diyl))dipyridine (DPEpe) and 1,4-diiodotetrafluorobenzene $\left(\mathrm{F}_{4} \mathrm{DIB}\right)$ single-crystal microrods and microtubes prepared via the solution eva- 


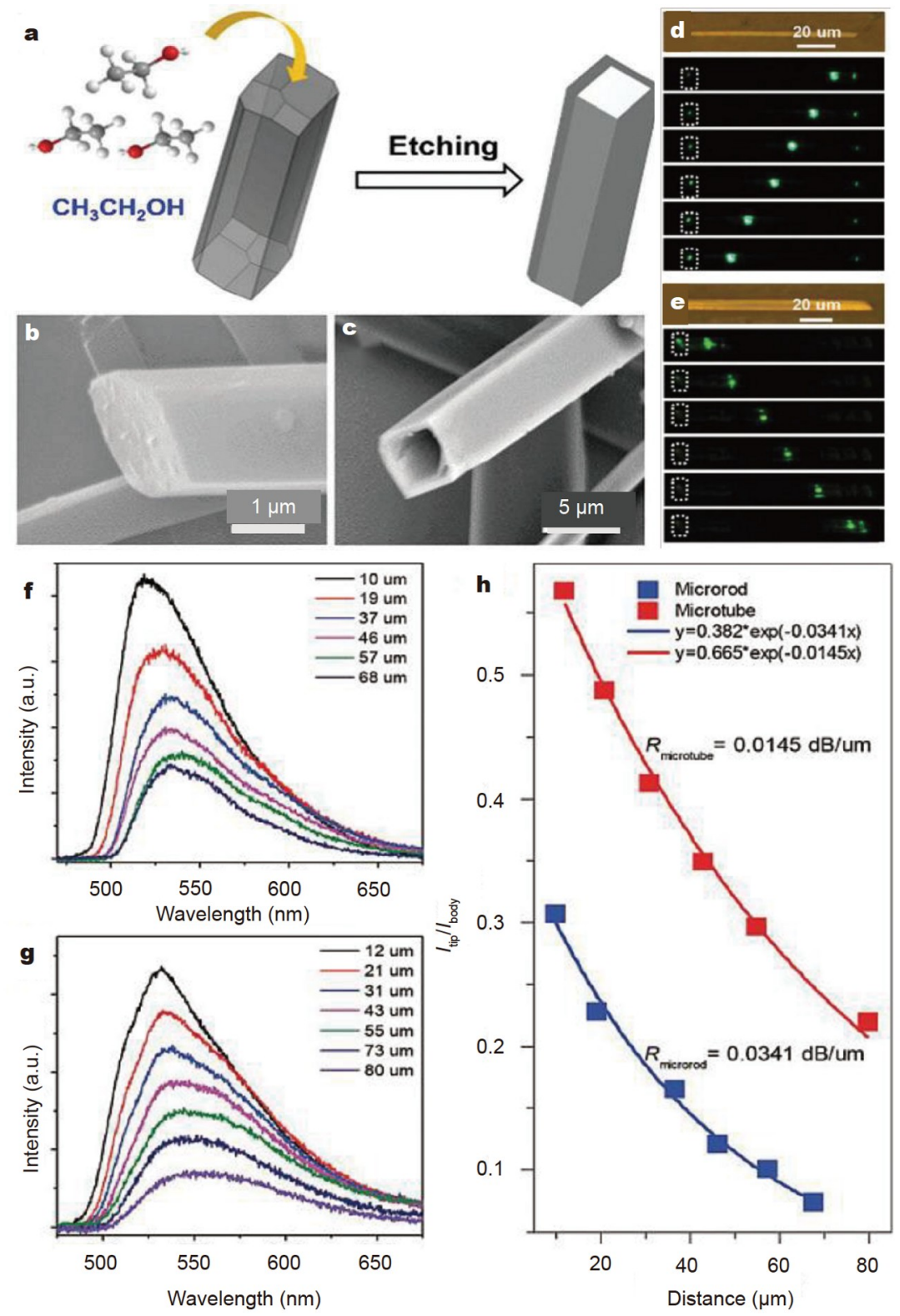

Figure 14 (a) Schematic diagram of the etching process of the microcrystal. (b, c) SEM images of the microrod and the microtube. (d, e) Bright-field and FM images of the microcrystals. (f, g) The $\mu$-PL spectra of the organic microcrystals. (h) Ratios of the $I_{\text {tip }} / I_{\text {body }}$ against the distance $D$ corresponding to (d) and (e). Reproduced with permission from Ref. [85]. Copyright 2018, Royal Society of Chemistry.

poration method (Fig. 14a-c) [85]. The FM images for DPEpe$\mathrm{F}_{4} \mathrm{DIB}$ microrods and microtubes exhibit that the photons are restricted in $2 \mathrm{D}$ and then travel in two opposite directions along the axes of the microstructure (Fig. 14d, e). Active optical waveguide phenomenon is observed by recording the PL intensity at the excitation position $\left(I_{\text {body }}\right)$ and emitting position ( $\left.I_{\text {tip }}\right)$ of the microstructure; the $I_{\text {tip }} / I_{\text {body }}$ ratio against the propagation distance shows an exponential decay (Fig. 14f, g). The optical loss coefficient $(R)$ of the microtube is $0.0145 \mathrm{~dB}_{\mu \mathrm{m}}^{-1}$

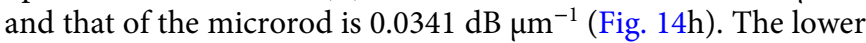
optical loss coefficient of the microtube is attributed to the $2 \mathrm{D}$ constraint of photons in the thin tube wall. Shape-dependent optical properties for organic micro/nanocrystals provide useful guidance for the designing and preparation of micro/nanoscale miniaturized functional optoelectronic devices.

\section{Polymorphsim approach}

The controlled manufacture of organic polymorphism with regular size and tunable luminescence properties provides an attractive approach for the development of organic optoelectronic devices, coherent light source, sensors and so on [71,127]. $\mathrm{Fu}$ and his colleagues [128] fabricated blue-emission or greenemission microribbons based on difluoroboron avobenzone $\left(\mathrm{BF}_{2} \mathrm{AVB}\right)$ through modulating cluster-mediated nucleation and subsequent growth processes. In the experiment, the aging time $t$ determines the molecule stacking models and the emission color of the microribbons in the first step as shown in Fig. 15a. In the first step, the $\mathrm{BF}_{2} \mathrm{AVB}$ clusters were generated when tetrahydrofuran (THF) solution was poured into the mixed poor solvents of cyclohexane and isopropanol, which served as nucleus for the subsequent self-assemble process. Within $3 \mathrm{~min}$, 
a
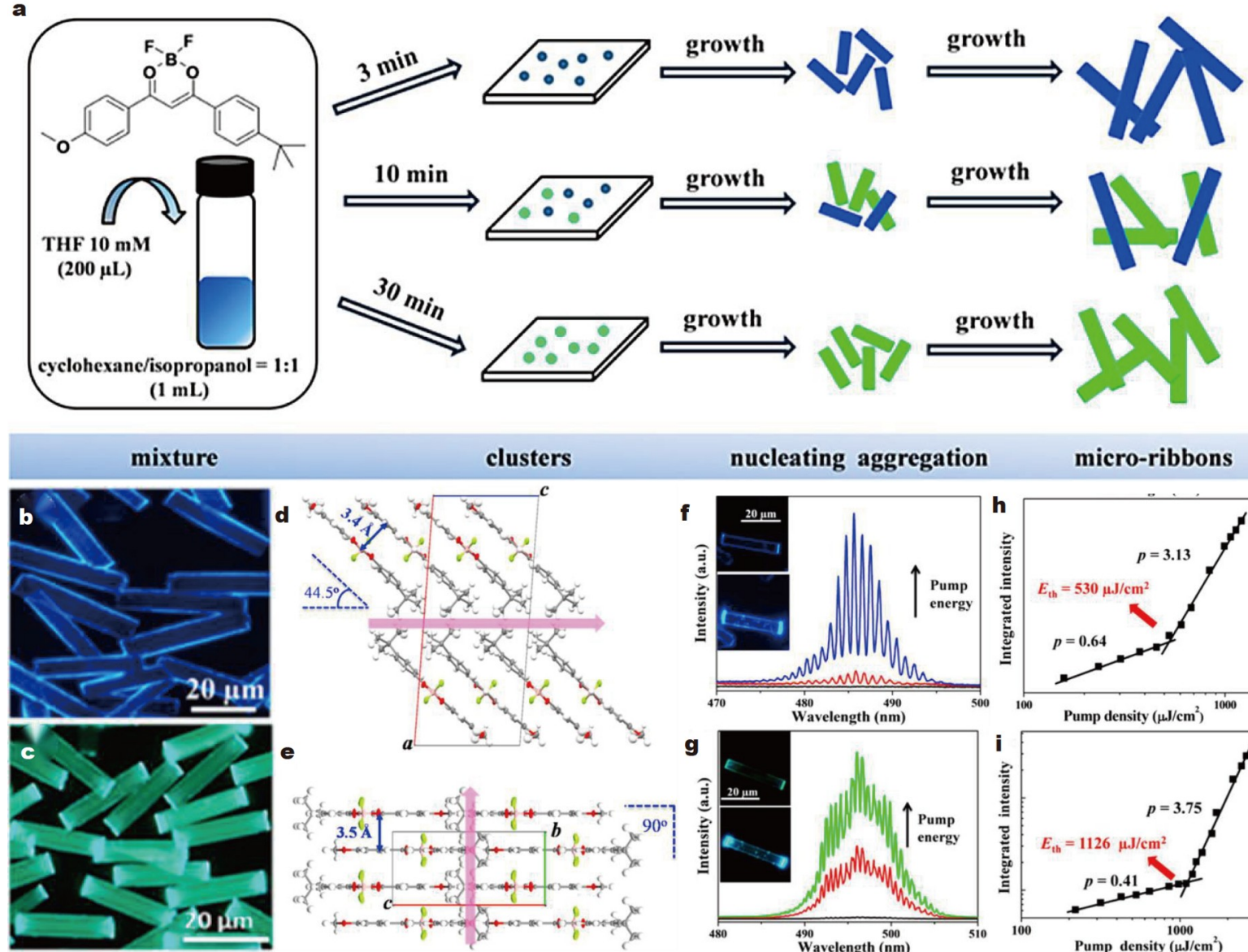

nucleating aggregation

micro-ribbons
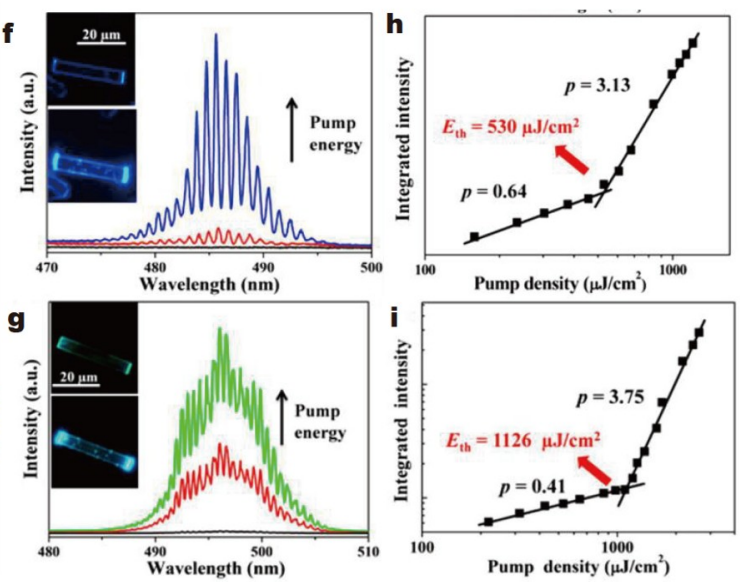

Figure 15 (a) Schematic illumination of the growth mechanism. (b, c) FM images of $\mathrm{BF}_{2} \mathrm{AVB}$ microrods excited with unfocused UV light. (d, e) Molecular stacking arrangement of $\mathrm{BF}_{2} \mathrm{AVB}$ molecules. (f, g) PL spectra of blue-emissive and green-emissive microrods. (h, i) Integrated area of the 0-1 PL peak as a function of pump density for blue-emission and green-emission microrods. Copyright 2018, Reproduced with permission from Ref. [128]. American Chemical Society.

the $\mathrm{BF}_{2} \mathrm{AVB}$ molecule can form loose monoclinic J-type clusters, showing a $13 \mathrm{~nm}$ red-shifted PL maximum at $437 \mathrm{~nm}$ compared with the solution PL peak (Fig. 15b, d). However, when the aging time exceeds $30 \mathrm{~min}$, closed packed orthorhombic H-type clusters of $\mathrm{BF}_{2} \mathrm{AVB}$ microribbons are formed, which exhibit greenemission (Fig. 15c, e).

In addition, when the aging time is $10 \mathrm{~min}$, both blue-emission and green-emission microribbons are observed, which indicates that the J-type aggregation changes to H-type aggregation with the extension of aging time. Fig. $15 f$ shows that when the pump intensity of $400 \mathrm{~nm}$ laser exceeds $535 \mu \mathrm{J} \mathrm{cm}^{-2}$, a group of peaks appear at the position of $0-1$ peak and the half-peak width of $0-1$ peak decreases sharply from $53 \mathrm{~nm}$ below the threshold to $5 \mathrm{~nm}$ above the threshold. From the intensity dependence characterization, it can be seen that the lasing threshold is located at $E_{\text {th }}=530 \mu \mathrm{J} \mathrm{cm}^{-2}$ as shown in Fig. $15 \mathrm{~h}$. As for the green-emission microribbons, the laser action is similar to that of the blue-emission microribbons (Fig. 15g). However, the lasing threshold is located at $1126 \mu \mathrm{J} \mathrm{cm}^{-2}$ (Fig. 15i), which is attributed to the photo inhibition of $0-0$ fluorescent transition of the green-emission H-type aggregation microribbons. Through this simple polymorphsim approach, J- and H-type microribbons are obtained, which exhibit different emission properties, good optical microresonator effects, and laser performance.
Additionally, all the molecular structures and the chemical formulas of the corresponding organic crystals mentioned above are summarized in Table 1.

\section{CONCLUTION AND OUTLOOK}

In this review, we firstly discussed the aggregation modes of organic crystals, mainly including the Kasha's model and the recently developed WFO coupling model. Subsequently, the morphology modulation strategies for the organic micro/nanocrystals have been summarized combined with the recent research progress, including side-chain construction, controlling supersaturation degree, solvent etching, and VD. Although many studies have focused on the shape modulation of organic micro/nanocrystals, trying to improve their controllability and pre-design ability, it is still a challenge to understand the basic growth mechanism. Finally, we have demonstrated the strategies for tuning the optical properties of organic micro/nanocrystals, including FRET routes, chemical reaction method, and excited state modulation, which influenced by the weak intermolecular interactions.

Up to now, many achievements about the fabrication of organic micro/nanocrystals have been made. However, the controllability of crystallization of organic molecules is quite challenging in terms of crystal phase, dimensions, geometrical 
Table 1 Molecular structures of the corresponding crystals

\begin{tabular}{|c|c|c|c|}
\hline Molecular structure & Chemical formula & Abbreviation & Ref. \\
\hline & $\left(2 Z, 2^{\prime} Z\right)-2,2^{\prime}$-(1,4-phenylene)bis(3-(4-butoxyphenyl)acrylonitrile) & DBDCS & {$[52]$} \\
\hline & 4-(4a,10a-dihydro- $10 H$-phenothiazin-10-yl)benzonitrile & $\mathrm{CzS}-\mathrm{CN}$ & {$[126]$} \\
\hline & 1,4-Bis $((E)$-4-methylstyryl)benzene & $p$-MSB & {$[66]$} \\
\hline & 1,4-Bis $((E)$-2-methylstyryl)benzene & $o-\mathrm{MSB}$ & {$[66]$} \\
\hline & 1-(6-(Dimethylamino)naphthalen-2-yl)ethan-1-one & $\mathrm{ADN}$ & {$[67]$} \\
\hline & 1-(6-(Methylamino)naphthalen-2-yl)ethan-1-one & $\mathrm{AMN}$ & {$[67]$} \\
\hline & 2,5,8,11-Tetra-tert-butylperylene & TBPe & {$[70]$} \\
\hline & $\begin{array}{c}\text { 1,4-Bis }\left(1,2^{\prime}: 6^{\prime}, 1^{\prime \prime} \text {-bis(3-butyl-1H-3,4,5-triazolyl)pyridin- }\right. \\
4^{\prime} \text {-yl)benzene }\end{array}$ & - & {$[80]$} \\
\hline & $\begin{array}{l}\text { (E)-3-(4-(dimethylamino)-2-methoxyphenyl)-1- } \\
\text { (1-hydroxynaphthalen-2-yl)prop-2-en-1-one }\end{array}$ & DMHP & {$[82]$} \\
\hline & $\begin{array}{l}\text { 4,40-((1E,10E)-(2,5-dimethoxy-1,4-phenylene)bis } \\
\text { (ethene-2,1-diyl))dipyridine }\end{array}$ & DPEpe & {$[83]$} \\
\hline & 1,4-Diiodotetrafluorobenzene & $\mathrm{F}_{4} \mathrm{DIB}$ & {$[83]$} \\
\hline & 9,10-Bis(phenylethynyl)anthracene & BPEA & {$[90]$} \\
\hline & $5,10,15,20$-Tetrakis( $p$-chlorophenyl)porphyrin & TCIPP & {$[97]$} \\
\hline & Perylene nanoparticles & PeNP & {$[103]$} \\
\hline & $\begin{array}{l}\text { 3-[4-(Dimethylamino)phenyl]-1-(2-hydroxyphenyl)prop- } \\
\text { 2-en-1-one }\end{array}$ & HDMAC & [128] \\
\hline & 1,4-Bis $((E)-2$-(3-methylpyridin-4-yl)vinyl)benzene & MSP & {$[117]$} \\
\hline & 1,2-Di(4-pyridyl)ethylene & trans-Bpe & {$[119]$} \\
\hline & 1,3,5-Trifluoro-2,4,6-triiodobenzene & IFB & {$[119]$} \\
\hline & 9,9'-(2,5-Dibromo-1,4-phenylene)bis( $9 H$-carbazole) & $\mathrm{PDBCz}$ & {$[123]$} \\
\hline & Difluoroboron avobenzone & $\mathrm{BF}_{2} \mathrm{AVB}$ & [125] \\
\hline
\end{tabular}


shape, and combination of components. To solve these problems, scientists can choose two or more organic materials that possess compatible molecular structures, sizes, and physicochemical properties, which enable the random mixing in their crystalline mixtures. Besides, another challenge for organic micro/nanocrystals is that the relationship between the molecular structure-aggregation behavior and micro/nanostructureoptical properties is still non-quantitative, which needs to be further established precisely. In future studies, the relationship between them can be investigated by changing the molecular staking modes, such as the substituent groups' effect and the cocrystals approach, which finally lead to the disparity in the performance of the miniature organic devices. Especially, organic micro/nanostructures are widely used in the photoelectric field, so it is necessary to investigate the controlled synthesis of the organic micro/nanocrystals with adjustable morphology and optical properties.

Organic microcrystals are fragile and unstable in the air or under other external environments, which is a significant drawback compared with inorganic or metal micro/nanostructures. Nevertheless, the solvent-resistant coating method which improves the solvent resistance of organic micro/nanocrystals would solve this problem to a large extent. Many efforts are needed to further investigate the photostability mechanism of organic microcrystals and improve their stability through the possible strategies. As we all know, there is indeed a long way to go for practical applications of the organic crystals. Due to the increasing interests in the organic micro/nanocrystals from the researchers around the world, we believe that organic crystals with tunable morphologies and optical properties will provide the platform for the realization of practical optoelectronic devices.

\section{Received 30 September 2021; accepted 27 October 2021;} published online 27 December 2021

1 Kuehne AJC, Gather MC. Organic lasers: Recent developments on materials, device geometries, and fabrication techniques. Chem Rev, 2016, 116: 12823-12864

2 Lauhon LJ, Gudiksen MS, Wang D, et al. Epitaxial core-shell and coremultishell nanowire heterostructures. Nature, 2002, 420: 57-61

3 Zhuo MP, He GP, Yuan Y, et al. Super-stacking self-assembly of organic topological heterostructures. CCS Chem, 2021, 3: 413-424

4 Ramanavicius S, Ramanavicius A. Conducting polymers in the design of biosensors and biofuel cells. Polymers, 2021, 13: 49

5 Uzunçar S, Meng L, Turner APF, et al. Processable and nanofibrous polyaniline:polystyrene-sulphonate (nano-PANI:PSS) for the fabrication of catalyst-free ammonium sensors and enzyme-coupled urea biosensors. Biosens Bioelectron, 2021, 171: 112725

6 Kong M, Jin P, Wei W, et al. Covalent organic frameworks (COF-300$\mathrm{AR}$ ) with unique catalytic performance in luminol chemiluminescence for sensitive detection of serotonin. Microchem J, 2021, 160: 105650

7 Wang Y, Gong Q, Miao Q. Structured and functionalized organic semiconductors for chemical and biological sensors based on organic field effect transistors. Mater Chem Front, 2020, 4: 3505-3520

8 Bala I, Singh N, Yadav RAK, et al. Room temperature perylene based columnar liquid crystals as solid-state fluorescent emitters in solutionprocessable organic light-emitting diodes. J Mater Chem C, 2020, 8: $12485-12494$

9 Tao Y, Peng S, Wang X, et al. Sequential self-assembly of 1D branched organic homostructures with optical logic gate function. Adv Funct Mater, 2018, 28: 1804915

10 Arulkashmir A, Jain B, John JC, et al. Chemically doped perylene diimide lamellae based field effect transistor with low operating vol- tage and high charge carrier mobility. Chem Commun, 2014, 50: 326328

11 Ko DH, Tumbleston JR, Zhang L, et al. Photonic crystal geometry for organic solar cells. Nano Lett, 2009, 9: 2742-2746

12 Steiner F, Lupton JM, Vogelsang J. Role of triplet-state shelving in organic photovoltaics: Single-chain aggregates of poly(3-hexylthiophene) versus mesoscopic multichain aggregates. J Am Chem Soc, 2017, 139: 9787-9790

13 Liao H, Chen M, Sun J, et al. Novel and asymmetric $S, N$-heterocyclics with fused six-membered rings for organic field effect transistor applications. J Mater Chem C, 2020, 8: 17083-17089

14 Kim FS, Ren G, Jenekhe SA. One-dimensional nanostructures of $\pi$ conjugated molecular systems: Assembly, properties, and applications from photovoltaics, sensors, and nanophotonics to nanoelectronics. Chem Mater, 2011, 23: 682-732

15 Cai K, Xie J, Zhao D. NIR J-aggregates of hydroazaheptacene tetraimides. J Am Chem Soc, 2014, 136: 28-31

16 Naren G, Hsu CW, Li S, et al. An all-photonic full color RGB system based on molecular photoswitches. Nat Commun, 2019, 10: 7

17 Yang L, Dou Y, Qin L, et al. A lanthanide-containing coordination polymer using tetraphenylethene-based linkers with selective $\mathrm{Fe}^{3+}$ sensing and efficient iodine adsorption activities. Inorg Chem, 2020, 59: $16644-16653$

18 Li Q, Li Z. Miracles of molecular uniting. Sci China Mater, 2020, 63: $177-184$

19 Li Q, Li Z. Molecular packing: Another key point for the performance of organic and polymeric optoelectronic materials. Acc Chem Res, 2020, 53: 962-973

20 Li Q, Li Z. The strong light-emission materials in the aggregated state: What happens from a single molecule to the collective group. Adv Sci, 2017, 4: 1600484

21 Würthner F, Kaiser TE, Saha-Möller CR. J-aggregates: From serendipitous discovery to supramolecular engineering of functional dye materials. Angew Chem Int Ed, 2011, 50: 3376-3410

22 Dong R, Zhou Y, Huang X, et al. Functional supramolecular polymers for biomedical applications. Adv Mater, 2015, 27: 498-526

23 Zhang Y, Zeng Q, Shen Y, et al. Electrochemical stability investigations and drug toxicity tests of electrolyte-gated organic field-effect transistors. ACS Appl Mater Interfaces, 2020, 12: 56216-56221

24 Shi YL, Zhuo MP, Wang XD, et al. Two-dimensional organic semiconductor crystals for photonics applications. ACS Appl Nano Mater, 2020, 3: 1080-1097

25 Wu JJ, Wang XD, Liao LS. Near-infrared solid-state lasers based on small organic molecules. ACS Photonics, 2019, 6: 2590-2599

26 Zhao F, Liu C, Sun Y, et al. Controlled self-assembly of triazatruxene overlength microwires for optical waveguide. Org Electron, 2019, 74 276-281

27 Min S, Dhamsaniya A, Zhang L, et al. Scale effect of a fluorescent waveguide in organic micromaterials: A case study based on coumarin microfibers. J Phys Chem Lett, 2019, 10: 5997-6002

28 Mukherjee A, Sakurai T, Seki S, et al. Ultrathin two dimensional (2D) supramolecular assembly and anisotropic conductivity of an amphiphilic naphthalene-diimide. Langmuir, 2020, 36: 13096-13103

29 Liu H, Shen L, Cao Z, et al. Covalently linked perylenetetracarboxylic diimide dimers and trimers with rigid "J-type" aggregation structure. Phys Chem Chem Phys, 2014, 16: 16399-16406

30 Jancy B, Asha SK. Hydrogen-bonding-induced conformational change from $\mathrm{J}$ to $\mathrm{H}$ aggregate in novel highly fluorescent liquid-crystalline perylenebisimides. Chem Mater, 2008, 20: 169-181

31 Hestand NJ, Spano FC. Interference between Coulombic and CTmediated couplings in molecular aggregates: $\mathrm{H}$ - to J-aggregate transformation in perylene-based $\pi$-stacks. J Chem Phys, 2015, 143: 244707

32 Bialas D, Brüning C, Schlosser F, et al. Exciton-vibrational couplings in homo- and heterodimer stacks of perylene bisimide dyes within cyclophanes: studies on absorption properties and theoretical analysis. Chem Eur J, 2016, 22: 15011-15018

33 Hestand NJ, Spano FC. Expanded theory of $\mathrm{H}-$ and J-molecular aggregates: The effects of vibronic coupling and intermolecular charge transfer. Chem Rev, 2018, 118: 7069-7163 
34 Zhang B, Lyskov I, Wilson LJ, et al. FRET-enhanced photoluminescence of perylene diimides by combining molecular aggregation and insulation. J Mater Chem C, 2020, 8: 8953-8961

35 Yao Y, Zhang L, Leydecker T, et al. Direct photolithography on molecular crystals for high performance organic optoelectronic devices. J Am Chem Soc, 2018, 140: 6984-6990

$36 \mathrm{Li}$ YX, Dong XM, Yu MN, et al. Enhanced emission in organic nanocrystals via asymmetrical design of spirocyclic aromatic hydrocarbons. Nanoscale, 2020, 12: 9964-9968

37 Huang J, Su Z, Huang M, et al. Spherical supramolecular structures constructed via chemically symmetric perylene bisimides: Beyond columnar assembly. Angew Chem Int Ed, 2020, 59: 18563-18571

38 Wang J, Kulago A, Browne WR, et al. Photoswitchable intramolecular H-stacking of perylenebisimide. J Am Chem Soc, 2010, 132: 41914196

39 Xie C, Yan F. Flexible photodetectors based on novel functional materials. Small, 2017, 13: 1701822

40 Kasha M. Energy transfer mechanisms and the molecular exciton model for molecular aggregates. Radiat Res, 1963, 20: 55-70

41 Hochstrasser RM, Kasha M. Application of the exciton model to mono-molecular lamellar systems. Photochem Photobiol, 1964, 3: 317-331

42 Kasha M, Rawls HR, Ashraf El-Bayoumi M. The exciton model in molecular spectroscopy. Pure Appl Chem, 1965, 11: 371-392

43 Hestand NJ, Spano FC. Molecular aggregate photophysics beyond the Kasha model: Novel design principles for organic materials. Acc Chem Res, 2017, 50: 341-350

44 Yamagata H, Pochas CM, Spano FC. Designing J- and H-aggregates through wave function overlap engineering: Applications to poly(3hexylthiophene). J Phys Chem B, 2012, 116: 14494-14503

45 Mukazhanova A, Trerayapiwat KJ, Mazaheripour A, et al. Accurate first-principles calculation of the vibronic spectrum of stacked perylene tetracarboxylic acid diimides. J Phys Chem A, 2020, 124: 30553063

46 Fink RF, Seibt J, Engel V, et al. Exciton trapping in $\pi$-conjugated materials: A quantum-chemistry-based protocol applied to perylene bisimide dye aggregates. J Am Chem Soc, 2008, 130: 12858-12859

47 Kaiser TE, Wang H, Stepanenko V, et al. Supramolecular construction of fluorescent J-aggregates based on hydrogen-bonded perylene dyes. Angew Chem Int Ed, 2007, 46: 5541-5544

48 Yagai S, Seki T, Karatsu T, et al. Transformation from H- to J-aggregated perylene bisimide dyes by complexation with cyanurates. Angew Chem Int Ed, 2008, 47: 3367-3371

49 Ghosh S, Li XQ, Stepanenko V, et al. Control of H- and J-type $\pi$ stacking by peripheral alkyl chains and self-sorting phenomena in perylene bisimide homo- and heteroaggregates. Chem Eur J, 2008, 14: 11343-11357

50 Würthner F, Saha-Möller CR, Fimmel B, et al. Perylene bisimide dye assemblies as archetype functional supramolecular materials. Chem Rev, 2016, 116: 962-1052

51 Oleson A, Zhu T, Dunn IS, et al. Perylene diimide-based $\mathrm{Hj}$ - and hJaggregates: The prospect of exciton band shape engineering in organic materials. J Phys Chem C, 2019, 123: 20567-20578

52 Yoon SJ, Chung JW, Gierschner J, et al. Multistimuli two-color luminescence switching via different slip-stacking of highly fluorescent molecular sheets. J Am Chem Soc, 2010, 132: 13675-13683

53 Yang J, Ren Z, Chen B, et al. Three polymorphs of one luminogen: How the molecular packing affects the RTP and AIE properties? J Mater Chem C, 2017, 5: 9242-9246

54 Wang J, Chai Z, Wang J, et al. Mechanoluminescence or room-temperature phosphorescence: Molecular packing-dependent emission response. Angew Chem Int Ed, 2019, 58: 17297-17302

55 Li ZZ, Liao LS, Wang XD. Controllable synthesis of organic microcrystals with tunable emission color and morphology based on molecular packing mode. Small, 2018, 14: 1702952

56 Uoyama H, Goushi K, Shizu K, et al. Highly efficient organic lightemitting diodes from delayed fluorescence. Nature, 2012, 492: 234238

57 Wong MY, Zysman-Colman E. Purely organic thermally activated delayed fluorescence materials for organic light-emitting diodes. Adv Mater, 2017, 29: 1605444

58 Dhondge AP, Huang YX, Lin T, et al. Benzodipyrrole-2,6-dione-3,7diylidenedimalononitrile derivatives for air-stable $n$-type organic fieldeffect transistors: Critical role of $\mathrm{N}$-alkyl substituent on device performance. J Org Chem, 2019, 84: 14061-14068

59 Shaikh DB, Ali Said A, Wang Z, et al. Influences of structural modification of naphthalenediimides with benzothiazole on organic fieldeffect transistor and non-fullerene perovskite solar cell characteristics. ACS Appl Mater Interfaces, 2019, 11: 44487-44500

60 Sundar VC, Zaumseil J, Podzorov V, et al. Elastomeric transistor stamps: reversible probing of charge transport in organic crystals Science, 2004, 303: 1644-1646

$61 \mathrm{Xu} \mathrm{Z}$, Liao Q, Shi Q, et al. Low-threshold nanolasers based on slabnanocrystals of $\mathrm{H}$-aggregated organic semiconductors. Adv Mater, 2012, 24: OP216-OP220

62 Yang $\mathrm{C}, \mathrm{Gu} \mathrm{L}, \mathrm{Ma} \mathrm{C}$, et al. Controllable co-assembly of organic micro/ nano heterostructures from fluorescent and phosphorescent molecules for dual anti-counterfeiting. Mater Horiz, 2019, 6: 984-989

63 Lan L, Liu H, Yu X, et al. Polymer-coated organic crystals with solvent-resistant capacity and optical waveguiding function. Angew Chem Int Ed, 2021, 60: 11283-11287

64 Ye X, Liu Y, Han Q, et al. Microspacing in-air sublimation growth of organic crystals. Chem Mater, 2018, 30: 412-420

65 Xue C, Jin S. Exceptionally strong electronic coupling in crystalline perylene diimides via tuning. Chem Mater, 2011, 23: 2689-2692

66 Zhao YS, Zhan P, Kim J, et al. Patterned growth of vertically aligned organic nanowire waveguide arrays. ACS Nano, 2010, 4: 1630-1636

67 Wang $\mathrm{X}, \mathrm{Li} \mathrm{H}, \mathrm{Wu} \mathrm{Y}$, et al. Tunable morphology of the self-assembled organic microcrystals for the efficient laser optical resonator by molecular modulation. J Am Chem Soc, 2014, 136: 16602-16608

68 Herr T, Brasch V, Jost JD, et al. Temporal solitons in optical microresonators. Nat Photon, 2014, 8: 145-152

69 Yao W, Yan Y, Xue L, et al. Controlling the structures and photonic properties of organic nanomaterials by molecular design. Angew Chem Int Ed, 2013, 52: 8713-8717

70 Horn D, Rieger J. Organic nanoparticles in the aqueous phaseTheory, experiment, and use. Angew Chem Int Ed, 2001, 40: 43304361

$71 \mathrm{Gu}$ J, Gao Y, Wu J, et al. Polymorph-dependent electrogenerated chemiluminescence of low-dimensional organic semiconductor structures for sensing. ACS Appl Mater Interfaces, 2017, 9: 8891-8899

72 Zhang X, Dong C, Zapien JA, et al. Polyhedral organic microcrystals: From cubes to rhombic dodecahedra. Angew Chem Int Ed, 2009, 48 9121-9123

73 Yu Y, Tao YC, Zou SN, et al. Organic heterostructures composed of one- and two-dimensional polymorphs for photonic applications. Sci China Chem, 2020, 63: 1477-1482

74 Wu S, Zhou B, Yan D. Recent advances on molecular crystalline luminescent materials for optical waveguides. Adv Opt Mater, 2021, 9: 2001768

75 Venkatakrishnarao D, Mohiddon MA, Chandrasekhar N, et al. Photonic microrods composed of photoswitchable molecules: Erasable heterostructure waveguides for tunable optical modulation. Adv Opt Mater, 2015, 3: 1035-1040

76 Apter B, Lapshina N, Handelman A, et al. Peptide nanophotonics: From optical waveguiding to precise medicine and multifunctional biochips. Small, 2018, 14: 1801147

77 Lee E, Huang Z, Ryu JH, et al. Rigid-flexible block molecules based on a laterally extended aromatic segment: Hierarchical assembly into single fibers, flat ribbons, and twisted ribbons. Chem Eur J, 2008, 14: 6957-6966

78 Nguyen TD, Glotzer SC. Switchable helical structures formed by the hierarchical self-assembly of laterally tethered nanorods. Small, 2009, 5: 2092-2098

79 Chen S, Yin H, Wu JJ, et al. Organic halogen-bonded co-crystals for optoelectronic applications. Sci China Mater, 2020, 63: 1613-1630

80 Zhao YS, Fu HB, Peng AD, et al. Construction and optoelectronic properties of organic one-dimensional nanostructures. Acc Chem Res, 


\section{0, 43: 409-418}

81 Ajayaghosh A, Chithra P, Varghese R. Self-assembly of tripodal squaraines: Cation-assisted expression of molecular chirality and change from spherical to helical morphology. Angew Chem Int Ed, 2007, 46: 230-233

82 Chandrasekhar N, Chandrasekar R. Reversibly shape-shifting organic optical waveguides: Formation of organic nanorings, nanotubes, and nanosheets. Angew Chem Int Ed, 2012, 51: 3556-3561

83 Huang Y, Wang Z, Chen Z, et al. Organic cocrystals: Beyond electrical conductivities and field-effect transistors (FETs). Angew Chem Int Ed, 2019, 58: 9696-9711

84 Zhuo MP, Zhang YX, Li ZZ, et al. Controlled synthesis of organic single-crystalline nanowires via the synergy approach of the bottomup/top-down processes. Nanoscale, 2018, 10: 5140-5147

85 Zhuo MP, Tao YC, Wang XD, et al. Rational synthesis of organic single-crystalline microrods and microtubes for efficient optical waveguides. J Mater Chem C, 2018, 6: 9594-9598

86 Pan ZW, Dai ZR, Wang ZL. Nanobelts of semiconducting oxides. Science, 2001, 291: 1947-1949

87 Qian F, Li Y, Gradečak S, et al. Gallium nitride-based nanowire radia heterostructures for nanophotonics. Nano Lett, 2004, 4: 1975-1979

88 Liu H, Li Y, Xiao S, et al. Synthesis of organic one-dimensional nanomaterials by solid-phase reaction. J Am Chem Soc, 2003, 125: 10794-10795

89 Zhang X, Jie J, Deng W, et al. Alignment and patterning of ordered small-molecule organic semiconductor micro-/nanocrystals for device applications. Adv Mater, 2016, 28: 2475-2503

90 Lee JK, Koh WK, Chae WS, et al. Novel synthesis of organic nanowires and their optical properties. Chem Commun, 2002, 138-139

91 Balzer F, Bordo VG, Simonsen AC, et al. Isolated hexaphenyl nanofibers as optical waveguides. Appl Phys Lett, 2003, 82: 10-12

$92 \mathrm{Wu} \mathrm{Y}$, Feng J, Jiang X, et al. Positioning and joining of organic singlecrystalline wires. Nat Commun, 2015, 6: 6737

93 Possin GE. A method for forming very small diameter wires. Rev Sci Instruments, 1970, 41: 772-774

94 Martin CR. Template synthesis of electronically conductive polymer nanostructures. Acc Chem Res, 1995, 28: 61-68

95 Parthasarathy RV, Martin CR. Synthesis of polymeric microcapsule arrays and their use for enzyme immobilization. Nature, 1994, 369: 298-301

96 Salman A, Sharif R, Javed K, et al. Controlled electrochemical synthesis and magnetic characterization of permalloy nanotubes. J Alloys Compd, 2020, 836: 155434

97 Pearson DH, Tonucci RJ, Bussmann KM, et al. Parallel patterning of mesoscopic ring arrays using nanochannel glass replica masks. Adv Mater, 1999, 11: 769-773

98 Kim TW, Park IS, Ryoo R. A synthetic route to ordered mesoporous carbon materials with graphitic pore walls. Angew Chem Int Ed, 2003, 42: $4375-4379$

99 Luo Z, Liu Y, Kang L, et al. Controllable nanonet assembly utilizing a pressure-difference method based on anodic aluminum oxide templates. Angew Chem Int Ed, 2008, 47: 8905-8908

100 Chen J, Herricks T, Geissler M, et al. Single-crystal nanowires of platinum can be synthesized by controlling the reaction rate of a polyol process. J Am Chem Soc, 2004, 126: 10854-10855

101 Chen M, Wu B, Yang J, et al. Small adsorbate-assisted shape control of Pd and Pt nanocrystals. Adv Mater, 2012, 24: 862-879

102 Li Y, Bastakoti BP, Malgras V, et al. Polymeric micelle assembly for the smart synthesis of mesoporous platinum nanospheres with tunable pore sizes. Angew Chem Int Ed, 2015, 54: 11073-11077

103 Yin $\mathrm{H}$, Wang $\mathrm{H}$, Li Z, et al. RNA micelles for the systemic delivery of anti-miRNA for cancer targeting and inhibition without ligand. ACS Nano, 2019, 13: 706-717

104 Yin J, Wang J, Li M, et al. Iodine ions mediated formation of monomorphic single-crystalline platinum nanoflowers. Chem Mater, 2012, 24: 2645-2654

105 Kang L, Wang Z, Cao Z, et al. Colloid chemical reaction route to the preparation of nearly monodispersed perylene nanoparticles: Sizetunable synthesis and three-dimensional self-organization. J Am
Chem Soc, 2007, 129: 7305-7312

106 Shinozuka Y, Matsuura M. Wannier exciton in quantum wells. Phys Rev B, 1983, 28: 4878-4881

107 Zhao YS, Fu H, Peng A, et al. Low-dimensional nanomaterials based on small organic molecules: Preparation and optoelectronic properties. Adv Mater, 2008, 20: 2859-2876

108 Scholes GD, Rumbles G. Excitons in nanoscale systems. Nat Mater 2006, 5: 683-696

109 Tao YC, Li ZZ, Wang XD, et al. Fluorescence/phosphorescence-conversion in self-assembled organic microcrystals. Chem Commun, 2018, 54: 5895-5898

110 Wang X, Liao Q, Lu X, et al. Shape-engineering of self-assembled organic single microcrystal as optical microresonator for laser applications. Sci Rep, 2014, 4: 7011

111 Scott BJ, Bartl MH, Wirnsberger G, et al. Energy transfer in dye-doped mesostructured composites. J Phys Chem A, 2003, 107: 5499-5502

112 Huang D, Wang C, Zou Y, et al. Bismuth interfacial doping of organic small molecules for high performance n-type thermoelectric materials. Angew Chem Int Ed, 2016, 55: 10672-10675

113 Cerdán L, Enciso E, Martín V, et al. FRET-assisted laser emission in colloidal suspensions of dye-doped latex nanoparticles. Nat Photon 2012, 6: 621-626

114 Li ZZ, Liang F, Zhuo MP, et al. White-emissive self-assembled organic microcrystals. Small, 2017, 13: 1604110

115 Bertorelle F, Lavabre D, Fery-Forgues S. Dendrimer-tuned formation of luminescent organic microcrystals. J Am Chem Soc, 2003, 125: 6244-6253

116 Bertorelle F, Rodrigues F, Fery-Forgues S. Dendrimer-tuned formation of fluorescent organic microcrystals. Influence of dye hydrophobicity and dendrimer charge. Langmuir, 2006, 22: 8523-8531

117 LaMer VK, Dinegar RH. Theory, production and mechanism of formation of monodispersed hydrosols. J Am Chem Soc, 1950, 72: 48474854

118 Bahmanyar S, Houk KN. The origin of stereoselectivity in prolinecatalyzed intramolecular aldol reactions. J Am Chem Soc, 2001, 123 12911-12912

119 Zhao JS, Ruan YB, Zhou R, et al. Memory of chirality in J-type aggregates of an achiral perylene dianhydride dye created in a chiral asymmetric catalytic synthesis. Chem Sci, 2011, 2: 937-944

120 Li ZZ, Wang XD, Liao LS. Luminescence-/morphology-modulation of organic microcrystals by a protonation process. J Mater Chem C, 2017, 5: 6661-6666

121 Boenigk D, Mootz D. Fluorides and fluoro acids. Part 18. The system pyridine-hydrogen fluoride at low temperatures. Formation and crystal structures of solid complexes with very strong NHF and FHF hydrogen bonding. J Am Chem Soc, 1988, 110: 2135-2139

122 Zhu W, Zheng R, Zhen Y, et al. Rational design of charge-transfer interactions in halogen-bonded co-crystals toward versatile solid-state optoelectronics. J Am Chem Soc, 2015, 137: 11038-11046

123 Zhang C, Yan Y, Jing YY, et al. One-dimensional organic photonic heterostructures: Rational construction and spatial engineering of excitonic emission. Adv Mater, 2012, 24: 1703-1708

124 Zhang C, Zhao YS, Yao J. Optical waveguides at micro/nanoscale based on functional small organic molecules. Phys Chem Chem Phys, 2011, 13: 9060

125 Mizuno H, Haku U, Marutani Y, et al. Single crystals of 5,5'-bis(4'methoxybiphenyl-4-yl)-2,2'-bithiophene for organic laser media. Adv Mater, 2012, 24: 5744-5749

126 Liu K, Huang K, Lv A, et al. Tunable microstructures of ultralong organic phosphorescence materials. Chem Commun, 2021, 57: 72767279

127 Liu Y, Hu H, Xu L, et al. Orientation-controlled 2D anisotropic and isotropic photon transport in co-crystal polymorph microplates. Angew Chem Int Ed, 2020, 59: 4456-4463

128 Liao Q, Wang XG, Lv S, et al. Cluster-mediated nucleation and growth of J- and H-type polymorphs of difluoroboron avobenzone for organic microribbon lasers. ACS Nano, 2018, 12: 5359-5367

Acknowledgements This work was supported by the National Natural 
Science Foundation of China (21971185), the Collaborative Innovation Center of Suzhou Nano Science and Technology (CIC-Nano), and the "111" Project of The State Administration of Foreign Experts Affairs of China.

Author contributions $\quad \mathrm{Ma} \mathrm{Y}$ and Li ZZ wrote the manuscript; Lin $\mathrm{H}$ and Wang XD developed the concept and revised the manuscript; Zhuo S prepared the figures; Chen S classified and analyzed the reference papers. All authors participated in the general discussion.

Conflict of interest The authors declare that they have no conflict of interest.

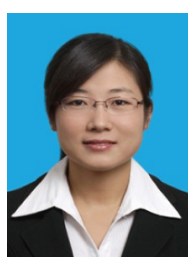

Hongtao Lin is an associate professor at the School of Chemistry and Chemical Engineering, Shandong University of Technology. She received her $\mathrm{PhD}$ degree from the Institute of Chemistry, Chinese Academy of Sciences in 2013 after she got her MSc degree (2010) from Lanzhou Institute of Chemical Physics, Chinese Academy of Sciences. Her research includes the design and synthesis of novel organic semiconductors, and the fabrication and characterization of organic optoelectronic devices.

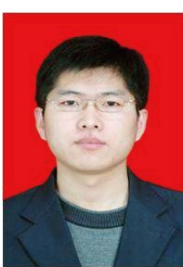

Shuhai Chen is a professor at the School of Chemistry and Chemical Engineering, Shandong University of Technology. In 2012, he received his $\mathrm{PhD}$ degree from Shanxi Institute of Coal Chemistry, Chinese Academy of Sciences. His research interests focus on molecular materials, polymer materials, and optoelectronic devices.

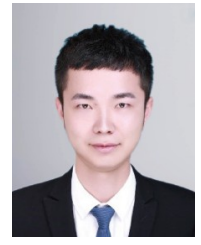

Xue-Dong Wang is a professor at the Institute of Functional Nano \& Soft Materials (FUNSOM), Soochow University. He received his Bachelor's degree in chemistry from Lanzhou University in 2011 and his $\mathrm{PhD}$ degree in physical chemistry from the Institute of Chemistry, Chinese Academy of Sciences in 2016. His research focuses on the fine synthesis of organic low-dimensional structures and the organic photonics including OSSLs and optical waveguides.

\section{基于有机微/纳米晶体形貌和光学性质的可控调制}

马英金 ${ }^{1 \dagger}$, 李治洲 ${ }^{2 \dagger}$, 萄红桃 ${ }^{1 *}$, 陈淑海 ${ }^{1 *}$, 禚淑萍 $^{1}$, 王雪东 ${ }^{2 *}$

摘要 基于有机小分子的低维有机微纳米晶体因在有机场效应晶体 管、电化学传感器、太阳能电池等领域的潜在应用而备受关注. 本文从 分子聚集模式、形貌调控、光学性质调控等方面综述了近十年来有机 微纳米晶体的研究进展. 首先介绍了分子聚集体模式对有机微/纳米晶 体物理化学性质(进而对其光学性质和形貌)的潜在影响, 接着总结了形 貌调控的多种方法和详细过程. 此外, 我们还探究了导致有机微/纳米晶 体不同光学性质的机理和调控方法. 最后, 我们从可控性和分子结构-聚 集行为-微/纳米结构-光学性质之间的不定量关系提出了有机微/纳米晶 体领域目前面临的挑战, 展望了具有可调形貌和光学特性的有机晶体应 用于实际光电器件中的前景. 这篇综述将促进有机微/纳米晶体的可控 合成及结构与光学性能关系的基础研究和实际应用方面的发展. 